\title{
ARTICLE Blocking FSH inhibits hepatic cholesterol biosynthesis and reduces serum cholesterol
}

Yanjing Guo ${ }^{1,2,3}$, Meng Zhao ${ }^{1,2,3}$, Tao Bo ${ }^{4}$, Shizhan Ma ${ }^{1,2,3}$, Zhongshang Yuan ${ }^{5}$, Wenbin Chen ${ }^{4}$, Zhao He ${ }^{2,3}, \mathrm{Xu} \mathrm{Hou}^{4}$, Jun Liu ${ }^{6}$, Zhenhai Zhang ${ }^{6}$, Qiang Zhu ${ }^{7}$, Qiangxiu Wang ${ }^{8}$, Xiaoyan Lin ${ }^{8}$, Zhongli Yang ${ }^{9}$, Min Cui ${ }^{9}$, Lu Liu ${ }^{1,2,3}$, Yujie Li ${ }^{1,2,3}, \mathrm{Chunxiao} \mathrm{Yu}^{1,2,3}$, Xiaoyi Qi ${ }^{1,2,3}$, Qian Wang ${ }^{1,2,3}$, Haiqing Zhang ${ }^{1,2,3}$, Qingbo Guan ${ }^{1,2,3}$, Lifang Zhao ${ }^{1,2,3}$, Shimeng Xuan ${ }^{1,2,3}$, Huili Yan ${ }^{1,2,3}$, Yanliang Lin ${ }^{4}$, $\mathrm{Li}_{\text {Wang }}{ }^{10}$, Qihang $\mathrm{Li}^{1,2,3}$, Yongfeng Song ${ }^{1,2,3,10}$, Ling Gao ${ }^{3,4}$ and Jiajun Zhao ${ }^{1,2,3}$

Menopause is associated with dyslipidemia and an increased risk of cardio-cerebrovascular disease. The classic view assumes that the underlying mechanism of dyslipidemia is attributed to an insufficiency of estrogen. In addition to a decrease in estrogen, circulating follicle-stimulating hormone (FSH) levels become elevated at menopause. In this study, we find that blocking FSH reduces serum cholesterol via inhibiting hepatic cholesterol biosynthesis. First, epidemiological results show that the serum FSH levels are positively correlated with the serum total cholesterol levels, even after adjustment by considering the effects of serum estrogen. In addition, the prevalence of hypercholesterolemia is significantly higher in peri-menopausal women than that in premenopausal women. Furthermore, we generated a mouse model of FSH elevation by intraperitoneally injecting exogenous FSH into ovariectomized (OVX) mice, in which a normal level of estrogen (E2) was maintained by exogenous supplementation. Consistently, the results indicate that FSH, independent of estrogen, increases the serum cholesterol level in this mouse model. Moreover, blocking FSH signaling by anti-FSH $\beta$ antibody or ablating the $F S H$ receptor (FSHR) gene could effectively prevent hypercholesterolemia induced by FSH injection or high-cholesterol diet feeding. Mechanistically, FSH, via binding to hepatic FSHRs, activates the Gi2a/ $\beta$-arrestin-2/Akt pathway and subsequently inhibits the binding of FoxO1 with the SREBP-2 promoter, thus preventing FoxO1 from repressing SREBP-2 gene transcription. This effect, in turn, results in the upregulation of SREBP-2, which drives HMGCR nascent transcription and de novo cholesterol biosynthesis, leading to the increase of cholesterol accumulation. This study uncovers that blocking FSH signaling might be a new strategy for treating hypercholesterolemia during menopause, particularly for women in peri-menopause characterized by FSH elevation only.

Cell Research (2019) 29:151-166; https://doi.org/10.1038/s41422-018-0123-6

\section{INTRODUCTION}

The life span of sexually mature females can be divided into three stages based on self-reported bleeding patterns, that is, premenopause, peri-menopause and post-menopause. ${ }^{1}$ During menopause, the prevalence of dyslipidemia also increases, which is the major risk factor of cardio-cerebrovascular disease. ${ }^{2-4}$ The classic view assumes that the underlying mechanism of dyslipidemia is attributed to an insufficiency of estrogen..$^{5}$ However, women during peri-menopause, at which time estrogen levels remain relatively unperturbed and only the FSH level increases, ${ }^{6}$ also suffer from a significant increase in total cholesterol (TC) and low-density lipoprotein cholesterol (LDL-C). ${ }^{2,7-9}$ This cholesterol accumulation begins 5 years before the final menstrual period (FMP), and substantially increases within 1 year of the FMP. ${ }^{7}$ Hormone replacement therapy (HRT) can decrease the serum cholesterol level, ${ }^{10,11}$ however, estrogen use can be associated with increased risks for breast cancer and coronary heart disease. ${ }^{5,12,13}$ The relatively poor alternatives for menopauserelated dyslipidemia therapies, particularly therapies for early and rapidly progressing cholesterol accumulation, makes the advent of new preventative strategies very desirable.

$\mathrm{FSH}$, a glycoprotein polypeptide hormone, is produced by the anterior pituitary gland, and is widely considered to function in the regulation of sex hormone synthesis in the gonads. ${ }^{14}$ During peri-menopause, because of compensation for failing ovaries, FSH increases before estrogen decreases. ${ }^{6,15}$ Strong correlations exist between rising serum FSH levels and osteoporosis and obesity after menopause. Blocking $\mathrm{FSH}$ reduced body fat levels and increased bone density in menopause. ${ }^{16-18}$ Furthermore, pre-menopausal women with high basal FSH levels (>7 IU/L)

\footnotetext{
${ }^{1}$ Department of Endocrinology, Shandong Provincial Hospital Affiliated to Shandong University, 250021 Jinan, Shandong, China; ${ }^{2}$ Shandong Key Laboratory of Endocrinology and Lipid Metabolism, 250021 Jinan, Shandong, China; ${ }^{3}$ Institute of Endocrinology and metabolism, Shandong Academy of Clinical Medicine, 250021 Jinan, Shandong, China; ${ }^{4}$ Scientific Center, Shandong Provincial Hospital Affiliated to Shandong University, 250021 Jinan, Shandong, China; ${ }^{5}$ Department of Biostatistics, School of Public Health, Shandong University, 250012 Jinan, Shandong, China; ${ }^{6}$ Department of Hepatobiliary Surgery, Shandong Provincial Hospital Affiliated to Shandong University, 250021 Jinan, Shandong, China; ${ }^{7}$ Department of Gastroenterology, Shandong Provincial Hospital Affiliated to Shandong University, 250021 Jinan, Shandong, China; ${ }^{8}$ Department of Pathology, Shandong Provincial Hospital Affiliated to Shandong University, 250021 Jinan, Shandong, China; ${ }^{9}$ Department of Gynecology, Shandong Provincial Hospital Affiliated to

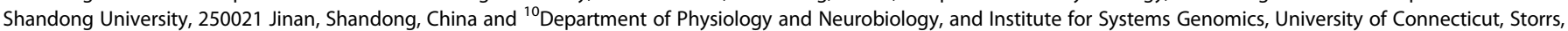
CT 06269, USA

Correspondence: Yongfeng Song (syf198506@163.com) or Ling Gao (linggao@sdu.edu.cn) or Jiajun Zhao (jjzhao@sdu.edu.cn, jjzhao@medmail.com.cn)
}

Received: 13 May 2018 Accepted: 15 November 2018

Published online: 17 December 2018 

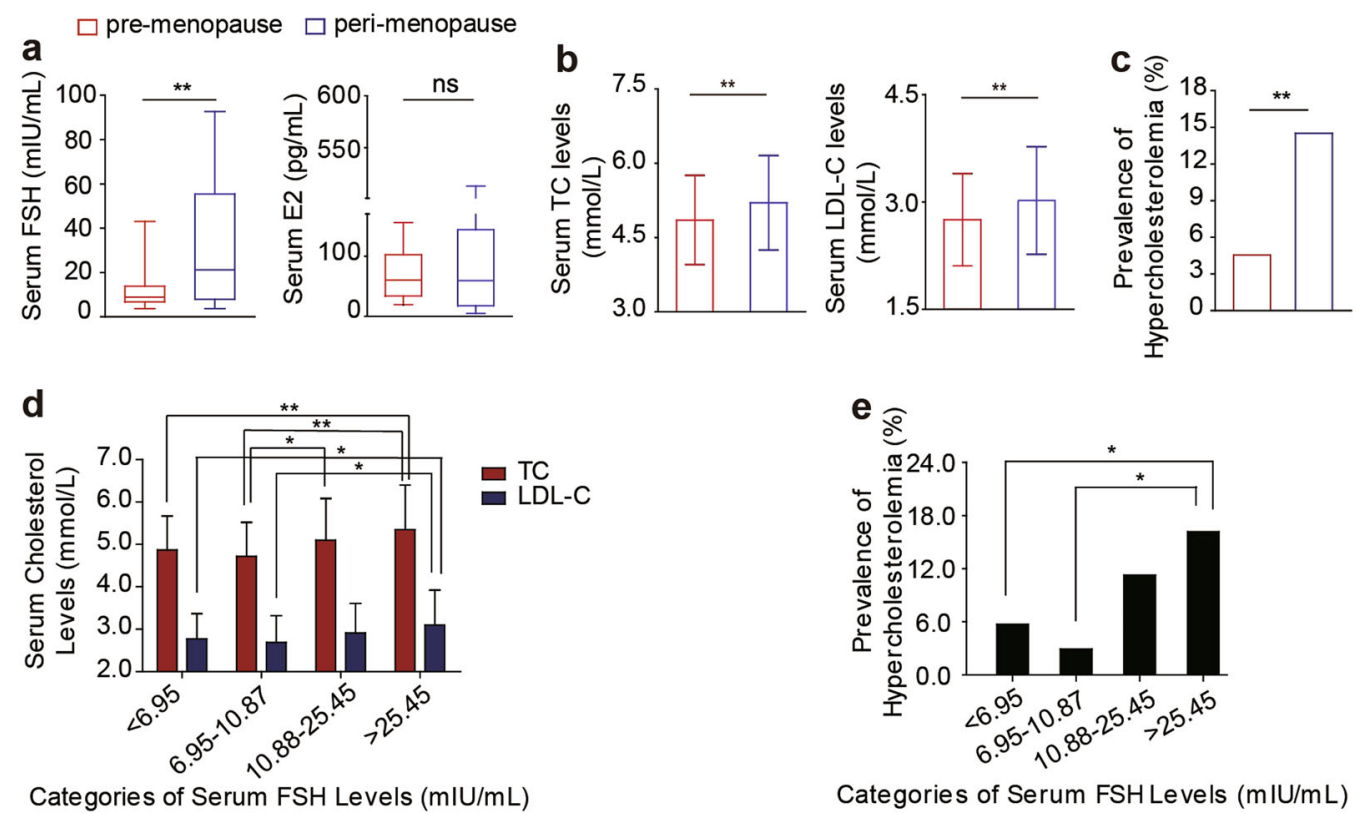

Fig. 1 Both serum cholesterol levels and the prevalence of hypercholesterolemia are higher in peri-menopausal women than in premenopausal ones. a Serum FSH and E2 levels. b Serum TC and LDL-C levels in the women with pre-menopause or peri-menopause. Data are presented as the mean \pm SD. c Prevalence of hypercholesterolemia in the recruited women. Data are presented as prevalence. $\mathbf{d}$ Cholesterol levels across the spectrum of serum FSH levels. Data are presented as the mean \pm SD. e Prevalence of hypercholesterolemia across the spectrum of serum FSH levels. Data are presented as prevalence. The statistical methods for a-e included the Mann-Whitney U-test, Student's $t$-test, chi-squared test, One-way ANOVA and chi-squared test, respectively. ${ }^{*} P<0.05,{ }^{*} P<0.01$; ns, not significant. TC, total cholesterol; LDL-C, low-density lipoprotein cholesterol

displayed greater cholesterol levels than those with basal FSH levels $\left(<7\right.$ IU/L). ${ }^{19}$ Reported by Song et al., post-menopausal women with a higher serum FSH ( $\geq 78.3 \mathrm{IU} / \mathrm{L})$ had higher serum TC and LDL-C levels than those with relatively lower FSH levels (40-78.3 IU/L), and ovariectomized mice had higher serum FSH and lipid levels and reduced hepatic LDL receptor expression. ${ }^{11}$ Together, all these data showed that FSH participated in the pathogenesis of menopause-related dyslipidemia. However, whether FSH signaling is a valuable therapeutic target for dyslipidemia remains largely unknown.

The function of FSH is mainly mediated through $\mathrm{FSH}$ receptors (FSHRs). ${ }^{14}$ FSHR is a transmembrane receptor that interacts with $\mathrm{FSH}$ and is a G protein-coupled receptor (GPCR). ${ }^{20}$ Published data demonstrate that FSHR couples with Gsa, which is involved in the differentiation and maturation of granulosa cells, ${ }^{20}$ or Gia, which regulates bone mass in osteoclasts as well as fat accumulation and redistribution in adipocytes. ${ }^{16,21}$ Arrestins are a small family of proteins, which are recruited by activated GPCRs through binding to GPCRs and act to uncouple the receptors from $G$ protein subunits. It has been reported that $\beta$-arrestin- 2 also acts as a multifunctional adapter following GPCR activation in metabolic processes. $^{22}$

The liver plays a pivotal role in maintaining cholesterol homeostasis, including cholesterol biosynthesis, uptake, conversion, transport and esterification. ${ }^{23}$ It is known that the biosynthesis of cholesterol starts from acetyl coenzyme A, in which 3hydroxy-3-methylglutaryl coenzyme $A$ reductase (HMGCR) acts as the rate-limiting enzyme. ${ }^{24}$ It has been proven that cyclic AMP response element-binding protein (CREB) and sterol regulatory element binding protein (SREBP)-2 are two important transcription factors that can bind with CAMP-responsive element (CRE) and sterol-response element (SRE), respectively, in the HMGCR promoter. ${ }^{25,26}$ SREBP-2 is an isoform of the SREBP family, which preferentially activates genes involved in cholesterol synthesis. ${ }^{27}$

Here, in women from an epidemiological investigation and in a mouse model with FSH elevation, we found a close correlation between serum FSH and cholesterol levels. The results indicate that elevated FSH alone can induce cholesterol biosynthesis via upregulating HMGCR expression and activity. Notably, blocking FSH signaling by anti-FSH $\beta$ antibody or ablating the Fshr gene could prevent cholesterol accumulation induced by elevated $\mathrm{FSH}$ or a high-cholesterol diet. This study provides new insights into hypercholesterolemia in menopausal females and shows that the inhibition of FSH signaling may be a new therapeutic strategy for treatment of hypercholesterolemia during menopause.

\section{RESULTS}

Serum FSH is positively correlated with TC levels and prevalence of hypercholesterolemia in women

The basic clinical characteristics of 154 pre-menopausal and 124 peri-menopausal female subjects are shown in Supplementary information, Table S1. Compared with the pre-menopausal women, the estrogen (E2) levels were similar in the perimenopausal females (Fig. 1a), however, the level of FSH, as well as the levels of TC and LDL-C (Fig. 1b, c) were all significantly increased. The range and distribution of the cholesterol levels are presented in Supplementary information, Fig. S1. Furthermore, the serum levels of TC and LDL-C and the prevalence of hypercholesterolemia gradually increased along with the increase of the serum FSH levels (Fig. 1d, e). Moreover, the serum FSH levels were positively correlated with the serum TC and LDL-C levels even after adjusting for E2 together with traditional confounding factors (Supplementary information, Table S2 and Table S3).

FSH increases serum and hepatic cholesterol accumulation in vivo and in vitro

To explore how FSH regulates cholesterol metabolism, it is necessary to establish an animal model to mimic the perimenopausal stage, which is characterized by elevated serum FSH levels. Due to a feedback regulation loop between FSH and estrogen ${ }^{14}$ and that decrease in estrogen levels can affect 
a

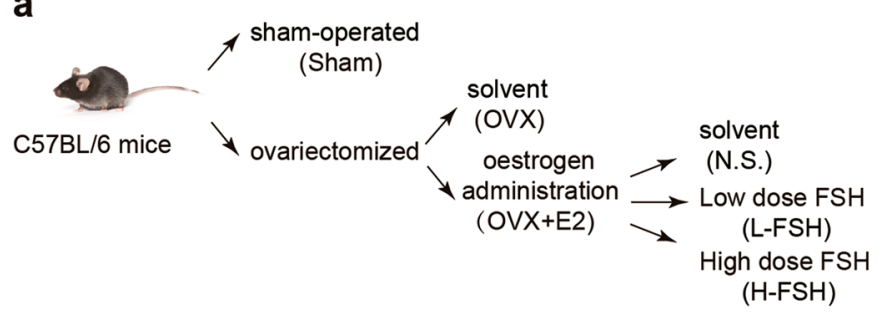

b

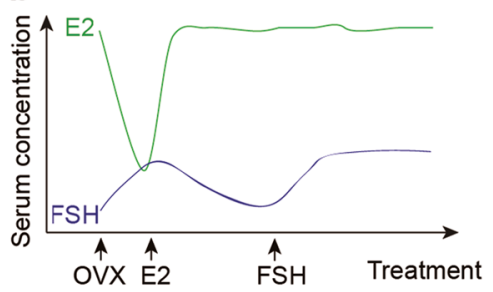

c

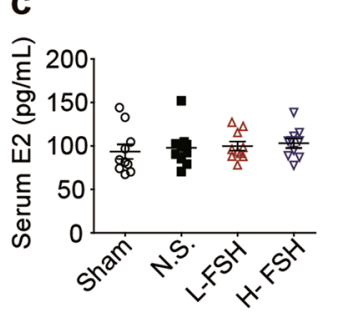

e

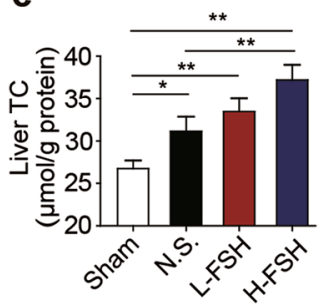

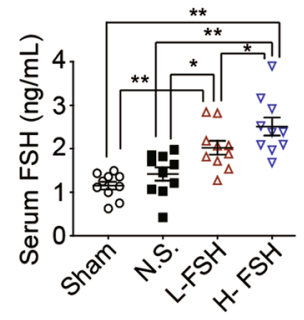

d
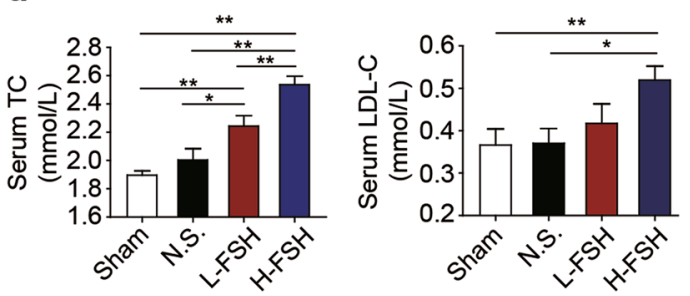

f

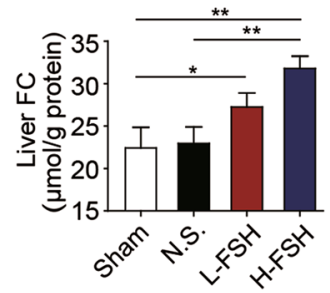

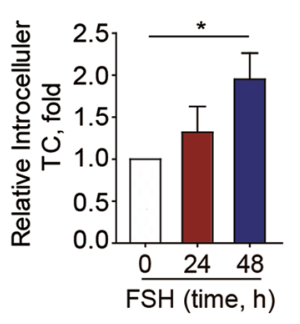

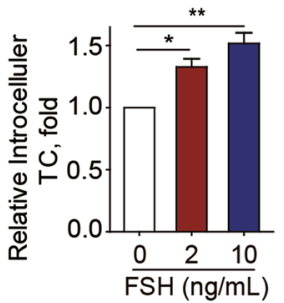

Fig. 2 FSH increases cholesterol accumulation in vivo and in vitro. a-e C57BL/6 mice were sham-operated (Sham) or ovariectomized (OVX), and then, the OVX mice were supplemented with estrogen (E2) to a normal level, named OVX + E2 mice, and then injected with solvent (normal saline, N.S.) or FSH (L-FSH represents low-dose, $15 \mathrm{IU} / \mathrm{kg}$ body weight per day; H-FSH represents high-dose, $30 \mathrm{IU} / \mathrm{kg}$ body weight per day) for 2 weeks ( $n=12$ for Sham group; $n=10$ per OVX groups). a Flow-chart. b Mimetic diagram of the serum E2 and FSH levels in the OVX mouse model. Arrows at the X-axis represent the starting points of the mice receiving different treatments. c ELISA analysis of serum E2 and FSH concentrations ( $n=10$ per group). d Serum TC and LDL-C levels. e Liver TC and FC contents. $\mathbf{f}$ Intracellular TC contents in HepG2 cells with FSH stimulation. Data represent three independent experiments. TC, total cholesterol; LDL-C, low-density lipoprotein cholesterol; FC, free cholesterol. Data are represented by the mean \pm SEM. One-way ANOVA was used for statistical analysis. ${ }^{*} P<0.05$ and ${ }^{* *} P<0.01$

cholesterol metabolism in the liver, $^{28}$ it is essential to maintain normal estrogen levels for excluding the effects of estrogen on cholesterol metabolism. Thus, we generated an ovariectomized (OVX) mouse model and maintained normal E2 level by exogenous supplementation of estrogen (OVX + E2). Under this condition, the mice were injected with exogenous FSH (Fig. 2a, b). Compared with the controls, the mice that received FSH demonstrated not only higher FSH levels but also normal E2 levels (Fig. 2c), which showed characteristics similar to those of peri-menopausal women. Moreover, the serum levels of TC and $\mathrm{LDL}-\mathrm{C}$, as well as the liver concentrations of TC and free cholesterol (FC), were significantly elevated following FSH treatment (Fig. 2d, e). Furthermore, we also measured the rate of hepatic very low-density lipoprotein (VLDL) production in FSHtreated OVX mice. We fasted mice overnight and treated them with poloxamer 407, a potent inhibitor of Lipoprotein lipase (LPL). The results showed that VLDL cholesterol production rate was significantly increased in the mice that received FSH (Supplementary information, Fig. S2). However, the body weights and serum aspartate transaminase (AST) levels did not significantly change (Supplementary information, Table S4). Similarly, FSH also increased the intracellular cholesterol content in vitro (Fig. 2f).

Blocking FSH reduces serum and hepatic cholesterol accumulation As elevated serum FSH levels are closely related to body cholesterol accumulation, we wondered whether blocking FSH could reverse the unfavorable results. First, we established an animal model that exhibited a low serum FSH level. Gonadotropin- releasing hormone analog $(\mathrm{GnRHa})$ inhibits the secretion of $\mathrm{FSH}$ in the body, ${ }^{29}$ which enabled us to mimic lower FSH levels in our mouse model (Fig. 3a, b). The results showed the mice that received $\mathrm{GnRHa}$ displayed significant decreases in the levels of FSH (Fig. 3c), serum TC, LDL-C, and the contents of liver TC and FC (Fig. 3d, e).

Furthermore, a polyclonal antibody against FSH was used to block FSH signaling. FSH consists of two dissimilar subunits, the a and $\beta$ subunits. ${ }^{30}$ The $\beta$-subunit of FSH $(\mathrm{FSH} \beta)$ is responsible for the interaction with FSHR. ${ }^{31}$ An anti-FSH $\beta$ antibody (FSHAb) has been proven to inhibit OVX-induced bone loss and body fat accumulation in mice. ${ }^{17,18,32}$ We demonstrated that when the FSHAb was added excessively, the effect of FSH in hepatocytes was nearly completely eliminated (Supplementary information, Fig. S3). To determine whether the antibody reversed FSHinduced cholesterol elevation in vivo, we used OVX mice with an exogenous injection of FSH/FSHAb in the presence of estrogen (Fig. 3f). As shown in Fig. 3g, h, blocking FSH could also effectively reduce the serum TC and LDL-C levels and the liver TC levels in OVX mice. There was no significant difference in the serum levels of E2 and FSH between antibody-treated and goat IgG-treated mice (Supplementary information, Table S5). More importantly, we also found that the suppression of FSH could decrease cholesterol under normal conditions. The FSHAb was used to block the interaction of FSH with the mouse FSHR by intraperitoneal injection for 8 weeks (Fig. 3i). The serum levels of E2 and FSH were not significantly different between the antibody-treated and IgG-treated mice (Supplementary 


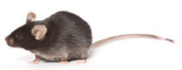

C57BL/6 mice

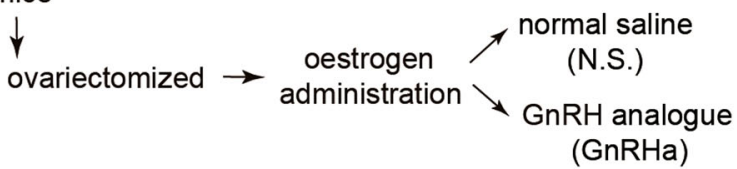

C

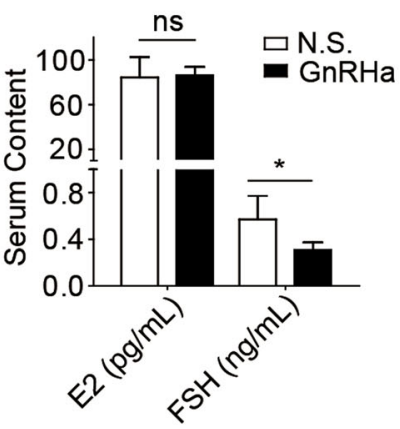

f

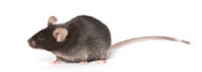

C57BL/6 mice

$\downarrow$

ovariectomized (OVX)

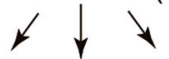

Normal saline IgG FSH $\beta$ antibody

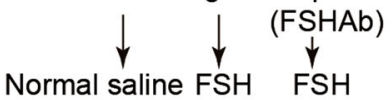

i

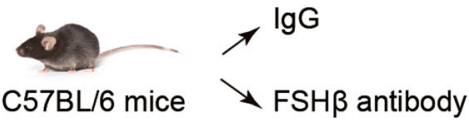

(FSHAb) b

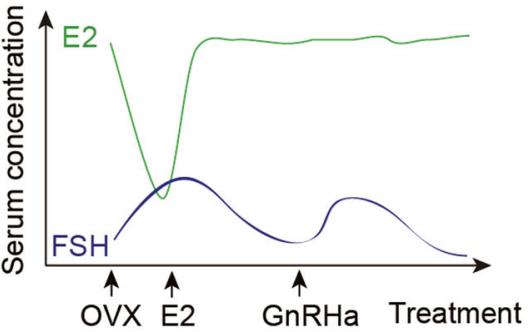

e

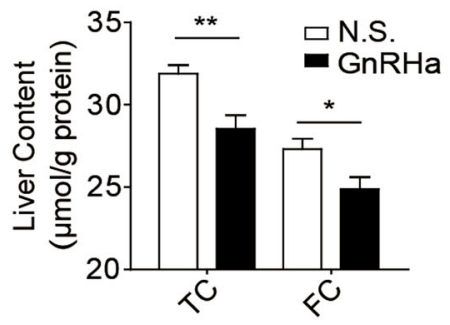

d

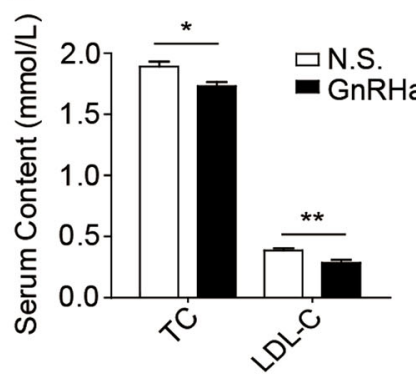

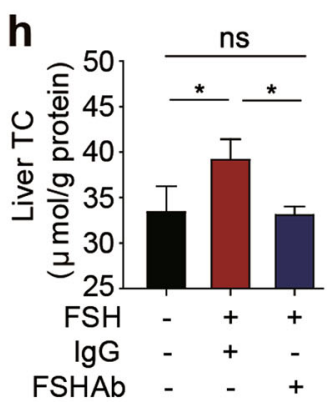

h
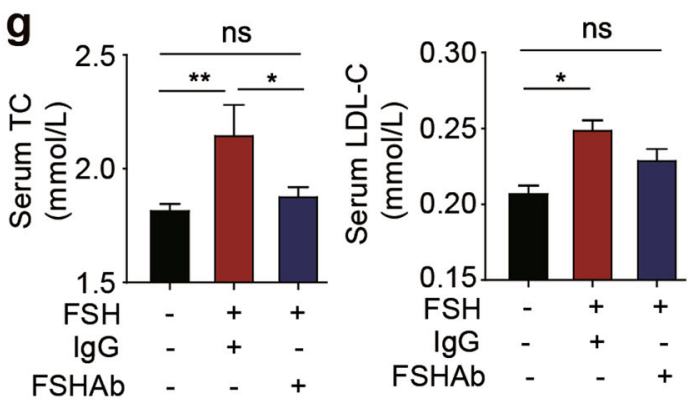

FSHAb - - +

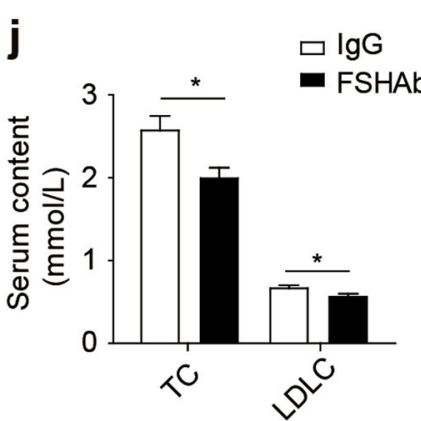

Fig. 3 Blocking FSH reduces serum and hepatic cholesterol accumulation. a-e E2-supplemented OVX mice received solvent (N.S.) or GnRH analog (GnRHa, $25 \mu \mathrm{g} / \mathrm{kg}$ body weight per day), which would lead to pituitary desensitization and to the inhibition of pituitary FSH secretion ( $n=6$ per group). a Workflow schematic. b Mimetic diagram of the changes following different treatments. Arrows at the X-axis represent the starting points of the mice receiving different treatments. c ELISA analysis of serum E2 and FSH concentrations. d Serum TC and LDL-C levels. e Liver TC and FC contents. f-h The OVX + E2 mice received an intraperitoneal injection of FSH $\beta$ antibody (FSHAb) or mouse IgG followed by treatments with FSH or normal saline (N.S.) for 4 weeks ( $n=6$ per group). $\mathbf{f}$ The flowchart. $\mathbf{g}$ The serum TC and LDL-C. $\mathbf{h}$ The liver TC. i-k The wild-type mice received an intraperitoneal injection of FSHAb (FSHAb group, $n=8$ ) or mouse IgG (IgG group, $n=10$ ) for 8 weeks. $\mathbf{i}$ The flowchart. $\mathbf{j}$ The serum TC and LDL-C. k The liver TC and FC. TC, total cholesterol; LDL-C, low-density lipoprotein cholesterol; FC, free cholesterol. Data are represented by the mean \pm SEM. One-way ANOVA or Student's $t$-test is used for statistical analysis. ${ }^{*} P<0.05$ and ${ }^{* *} P<$ $0.01 ; \mathrm{ns}$, not significant

information, Table S6), as previously indicated. ${ }^{17}$ However, the mice that received antibody showed significant decreases in the serum cholesterol levels and hepatic cholesterol content (Fig. 3j, k). These data suggest that blocking FSH may inhibit cholesterol metabolism and reduce body cholesterol in both physiological and pathological states.
Liver expresses functional FSHR

The function of FSH is mainly mediated through FSHR. In addition, as the liver is the chief organ for cholesterol metabolism in humans, we sought to determine whether there was functional FSHR expression in the liver. We designed four pairs of primers to cover different exons and domains of FSHR for humans and mice 
a

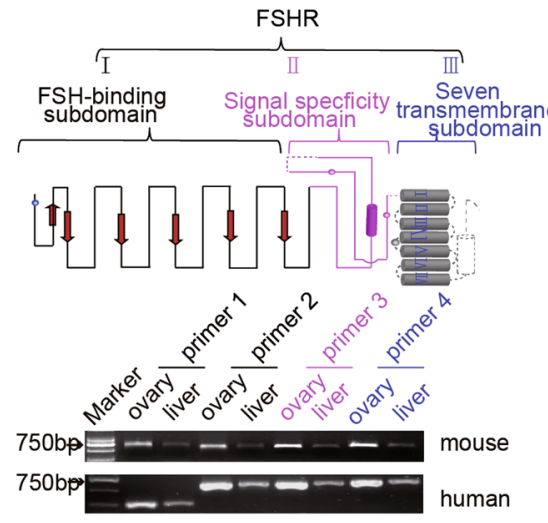

d

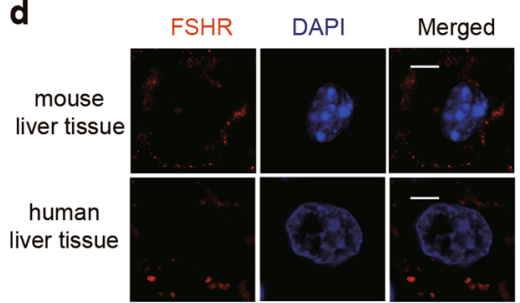

b

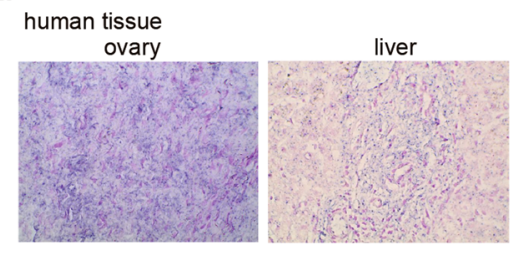

C

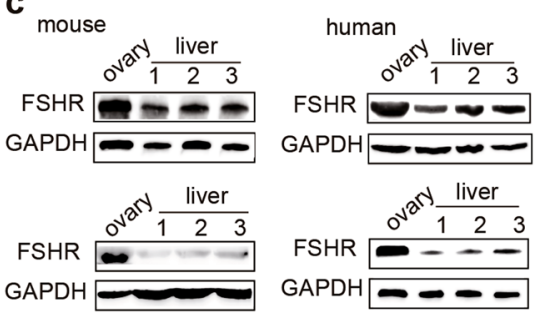

e

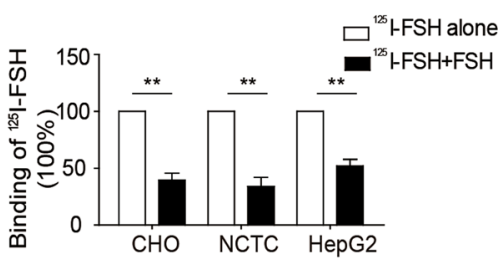

Fig. 4 Functional FSH receptors (FSHRs) are localized to the hepatocyte surface. a (Up) Primers were designed depending on different domains (I, II and III) of FSHR. (Down) Representative RT-PCR bands of FSHR from livers of human or mouse are shown, with RT-PCRs using ovary tissue as positive controls. b FSHR in situ hybridization images of the human ovary and liver. Cell nuclei are stained by nuclear fast red, and the blue granules indicate FSHR RNA. c Representative Western blotting results show the bands of mouse and human FSHR proteins using antibodies corresponding to regions within (Up) the C-terminus, or (Down) N-terminus of FSHR, $90 \mu \mathrm{g}$ protein per lane. d Representative super-resolution images of immunostaining of FSHR (red) and DAPI (blue) staining of nuclei in mouse and human liver tissues. Scale bars, 5 um. e FSH-FSHR binding assay in mouse (NCTC1469) and human (HepG2) hepatocytes, as well as in positive control (ovary cells, CHO). Data represent no less than three independent experiments. Data are shown as the mean \pm SEM. Student's $t$-test is used for statistical analysis. ${ }^{* *} P<$ 0.01

(Supplementary information, Table S7). Using human and mouse ovaries as the positive controls, we found the expected bands of FSHR in human and mouse livers by RT-PCR (Fig. 4a). The sequences of FSHR in both human and mouse liver tissues were consistent with the published mRNA sequences of FSHR derived from their respective positive controls (mouse FSHR, NM_013523.3; human FSHR, NM_181446.2) (Supplementary information, Figs. S4 and S5). In situ hybridization data showed that FSHR was expressed in human liver tissues (Fig. 4b). We found that FSHR protein was expressed in the liver by immunoblotting with different types of antibodies, which recognized the regions at either C-terminus or N-terminus of FSHR (Fig. 4c). To determine the cellular localization of FSHR, immunofluorescent staining was performed, and the results showed that FSHR was localized on the plasmalemma of hepatocytes (Fig. $4 \mathrm{~d}$ and Supplementary information, Movie S1). Using a competitive binding assay, we proved that both the human hepatocyte cell line HepG2 and the mouse hepatocyte cell line NCTC 1469, as well as CHO cells as a positive control, specifically bound ${ }^{125} \mathrm{I}-\mathrm{FSH}$ (Fig. 4e). Together, these findings indicate that there is functional FSHR expressed on the surface of hepatocytes.

Fshr deficiency resists hypercholesterolemia induced by $\mathrm{FSH}$ or high-cholesterol diet

FSHR acts as a gate keeper in the transmission of FSH signaling. To determine the extent of the contribution of FSHR to cholesterol accumulation in vivo, we performed ovariectomy operations in both $\mathrm{Fshr}^{-/-}$mice and $\mathrm{Fshr}^{+/+}$littermates, and all mice were subsequently injected with FSH and supplemented with E2 (Fig. 5a). Compared with their Fshr ${ }^{+/+}$littermates, the $\mathrm{Fshr}^{-1-}$ mice had similar serum E2 and FSH levels (Supplementary information, Table S8); however, the serum TC and LDL-C levels, as well as the liver TC and FC contents, were significantly reduced in the $\mathrm{Fshr}^{-1-}$ mice (Fig. 5b, c). Furthermore, FSH increased the intracellular TC levels in primary hepatocytes from $\mathrm{Fshr}^{+/+}$mice, which was not observed for $\mathrm{Fshr}^{-1-}$ mice (Fig. 5d). Moreover, to determine the specific function of FSHR in the liver, an adenovirus-mediated shRNA was used to specifically silence the expression of the hepatic Fshr gene via tail vein injection in OVX +E2 mice (Supplementary information, Fig. S6a). The results showed that the effects of FSH on cholesterol were reversed when the Fshr gene was silenced (Supplementary information, Fig. S6b). To explore the regulation of FSH on cholesterol during the postmenopausal stage, we assessed the serum cholesterol levels in OVX mice without exogenous E2 supplementation in $\mathrm{Fshr}^{-1-}$ mice and $\mathrm{Fshr}^{+/+}$littermates, and the results showed that Fshr-deficient mice had significantly lower levels of serum cholesterol than $\mathrm{Fshr}^{+/+}$mice (Supplementary information, Fig. S6c). All the results suggest that Fshr deficiency inhibits hepatic cholesterol accumulation and reduces serum cholesterol levels in both the perimenopausal stage and post-menopausal stage.

To clarify whether Fshr-deficiency has a protective effect on hypercholesterolemia, we established a hypercholesterolemic mouse model via high-cholesterol diet (HC) feeding (Fig. 5e). The $\mathrm{Fshr}^{-/-}$mice were supplied with dietary estrogen after weaning and maintained serum E2 levels similar to those of their $\mathrm{Fshr}^{+/+}$ littermates (Supplementary information, Table S9). In the $\mathrm{Fshr}^{+/+}$ mice, HC feeding significantly increased the serum TC and LDL-C levels and the liver TC and FC contents as well as the hepatic protein levels of HMGCR. However, the effect of HC feeding was 
a

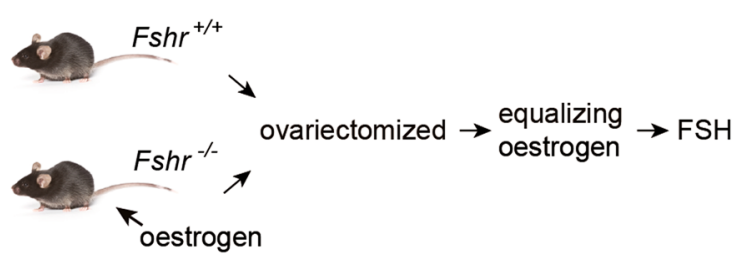

C
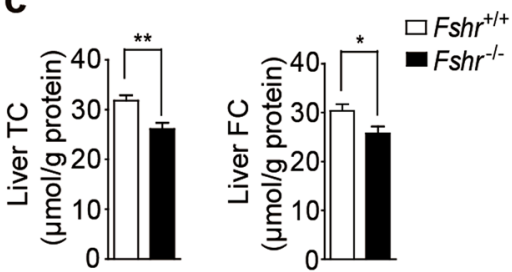

b

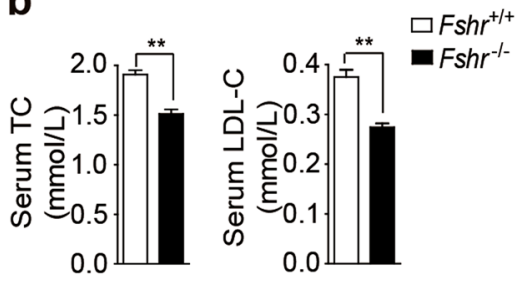

d

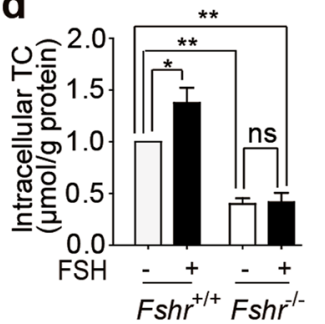

e

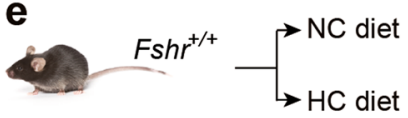

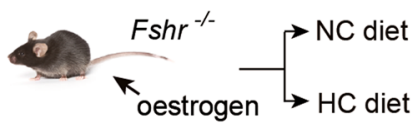

h

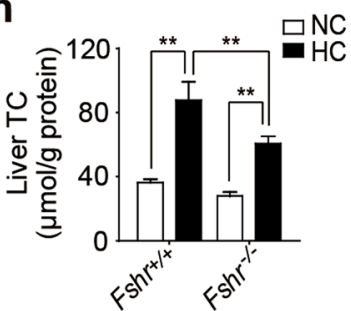

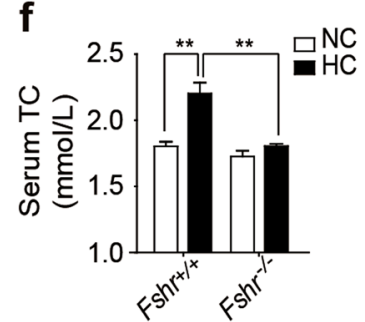

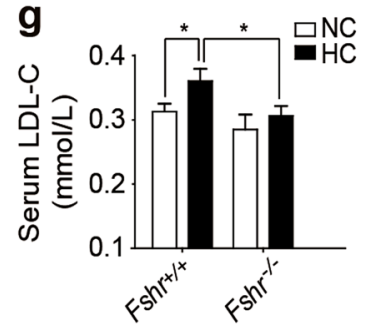

i

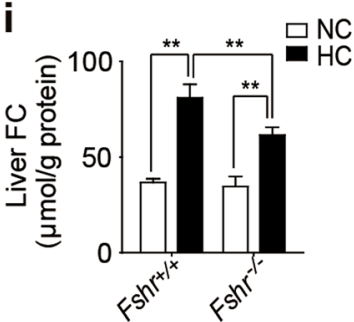

Fig. 5 Fshr deficiency resists hypercholesterolemia induced by FSH or high-cholesterol diet. a-c Ovariectomized $\mathrm{Fshr}^{-/-}$and $\mathrm{Fshr}^{+/+}$mice received FSH treatment for 2 weeks after equalizing their serum estrogen levels. $n=10$ per group. a The flowchart. $\mathbf{b}$ Analysis of serum TC and LDL-C levels, c liver TC and FC contents. $\mathbf{d}$ Intracellular TC contents were measured in mouse primary hepatocytes from $\mathrm{Fshr}^{-1-}$ mice and $\mathrm{Fshr}^{+/+}$ littermates treated with FSH $(10 \mathrm{ng} / \mathrm{mL})$, respectively. e-i The $\mathrm{Fshr}^{-/-}$mice and Fshr ${ }^{+/+}$littermates were fed with a normal cholesterol (NC) diet or high-cholesterol (HC) diet, and serum estrogen levels in all Fshr-- mice were normalized, $n=4$ for Fshr $r^{-1-}$ mice with $\mathrm{NC}$ diet; $n=8 \mathrm{for}$ other three groups. e The flowchart. f Serum TC and $\mathbf{g}$ serum LDL-C levels and $\mathbf{h}$ liver TC and $\mathbf{i}$ liver FC content. TC, total cholesterol; LDL-C, lowdensity lipoprotein cholesterol; FC, free cholesterol. Data are represented by the mean \pm SEM. One-way ANOVA or Student's $t$-test was used for statistical analysis. ${ }^{*} P<0.05,{ }^{*} P<0.01 ;$ ns, not significant

partially inhibited in the $\mathrm{Fshr}^{-/-}$mice (E2-supplemented) (Fig. 5f-i), which suggested that Fshr-deficiency may prevent highcholesterol diet-induced hypercholesterolemia.

FSH enhances nascent transcription of HMGCR and de novo cholesterol biosynthesis

Given that the liver plays a central role in lipid metabolism, ${ }^{23,33}$ we performed iTRAQ-based quantitative proteomic analysis to investigate hepatic proteome alterations and found significant differences in the sterol metabolic process in the comparison of the FSH-treated mice with the control mice (Fig. 6a). It is well known that cholesterol is the most important type of steroid in mammals. To elucidate the potential mechanism underlying the role of FSH in cholesterol metabolism, we used a RT-PCR array to scan 84 key genes involved in cholesterol metabolism, and the results showed significant changes in the genes related to cholesterol biosynthesis, transport, and esterification in the FSHtreated mice (Supplementary information, Table S10).
Furthermore, the expression of main molecules related to cholesterol metabolism was confirmed by $\mathrm{qPCR}$ and Western blotting. The expression of HMGCR, the rate-limiting enzyme in cholesterol biosynthesis, was significantly increased in the FSHtreated mice; however, no significant differences were observed for proteins related to cholesterol conversion, uptake, transport and esterification (Fig. 6b, c). Moreover, live image analysis indicated an increase in $H M G C R$ transcription in the mice treated with adenovirus that contained an HMGCR luciferase reporter plasmid (Ad-HMGCR/luc) followed by FSH injection (Fig. 6d). Actually, HMGCR activity is instrumental in controlling de novo cholesterol biosynthesis. ${ }^{34}$ Moreover, both the activity of HMGCR in vivo and the rate of hepatic de novo cholesterol biosynthesis also increased in a dose-dependent manner with FSH treatment (Fig. 6e). Using the run-on assay, we found that FSH increased the nascent transcription of HMGCR in hepatocytes (Fig. 6f). FSH also induced HMGCR expression in mouse primary hepatocytes and human HepG2 cells (Fig. $6 \mathrm{~g}-\mathrm{i}$ ). Actinomycin D, a transcriptional 
a

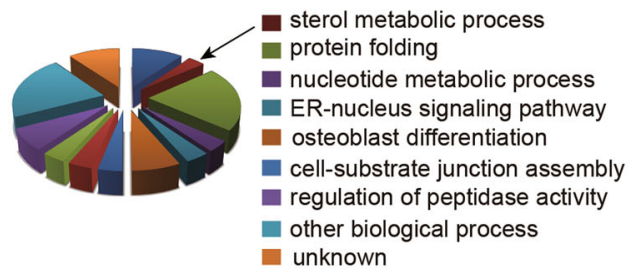

b

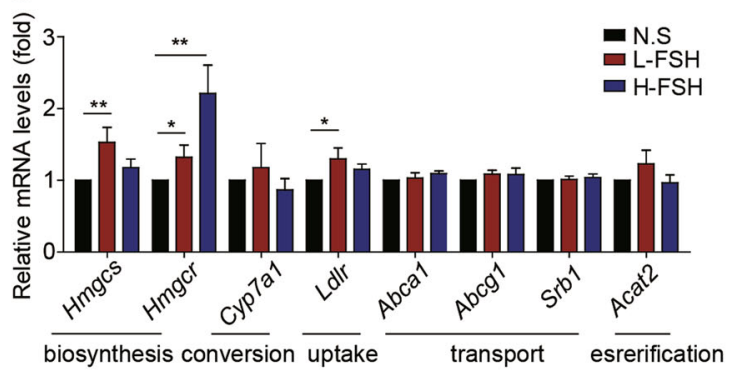

C

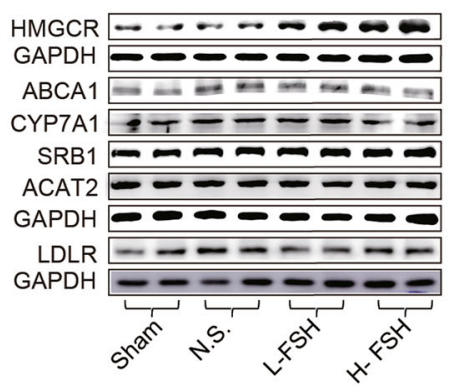

g

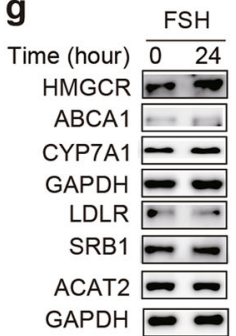

h

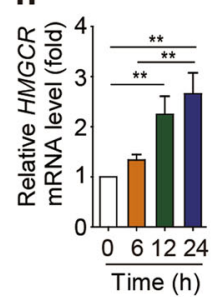

d

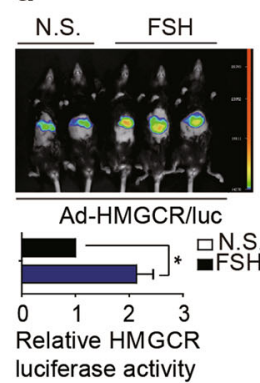

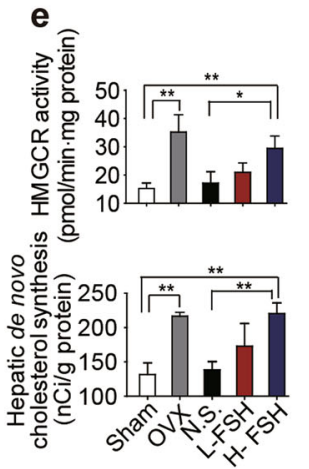

f

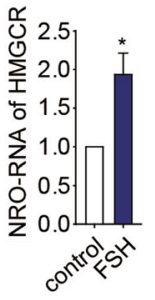

i

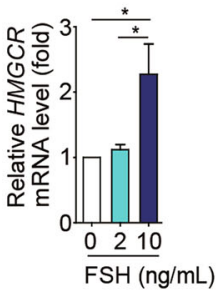

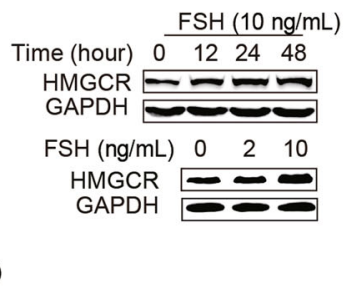

j

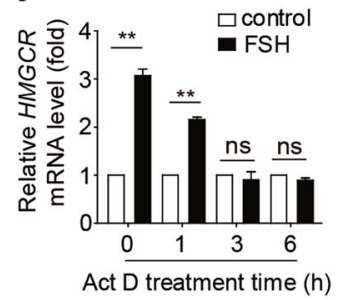

Fig. 6 FSH enhances nascent transcription of HMGCR and hepatic de novo cholesterol biosynthesis. a-e C57BL/6 mice were sham-operated (Sham) or ovariectomized (OVX), and then, the OVX mice were injected with solvent (normal saline, N.S.) or FSH (L-FSH represents low-dose, $15 \mathrm{lU} / \mathrm{kg}$ body weight per day; H-FSH represents high-dose, $30 \mathrm{lU} / \mathrm{kg}$ body weight per day), with estrogen (E2) maintained at normal levels. a The iTRAQ-based quantitative proteomic analysis of liver tissues compared the H-FSH group to the N.S. group ( $n=6$ per group). $\mathbf{b}$ mRNA levels and c Protein levels of molecules related to cholesterol metabolism in the liver $(n=10$ for the N.S. and H-FSH groups, $n=8$ for the L-FSH group). d Live imaging of hepatic HMGCR-luciferase (Ad-HMGCR/luc) activity in OVX +E2 mice after injection of Ad-HMGCR/luc via tail vein followed by injection of solvent (N.S.) or FSH (30 IU/kg) ( $n=6$ per group). e (Up) Detection of the in vivo activity of hepatic HMGCR, the ratelimiting enzyme in cholesterol biosynthesis, using the HPLC-MS/MS method ( $n=5$ for Sham group, $n=6$ for other groups). (Down) Hepatic de novo cholesterol biosynthesis was measured by the amount of $\left[1-{ }^{14} \mathrm{C}\right]$-acetate incorporated into sterols per gram of protein in the liver tissue ( $n=6$ for Sham, N.S and L-FSH groups; $n=4$ for OVX group; $n=5$ for H-FSH group). $f$ The nascent transcription of HMGCR in HepG2 cells treated with FSH $(10 \mathrm{ng} / \mathrm{mL})$ by run-on assay. $\mathbf{g}$ Representative immunoblotting of molecules related to cholesterol metabolism in mouse primary hepatocytes treated with FSH (10 ng/mL). $\mathbf{h}-\mathbf{j}$ Experiments were performed in the HepG2 cell line. $\mathbf{h}$ qPCR analysis of HMGCR in HepG2 cells treated with different FSH doses and for different time periods. i Representative Western blotting analysis of HMGCR in HepG2 cells treated with FSH. j qPCR analysis of the HMGCR mRNA level in HepG2 cells treated with $7.5 \mathrm{ng} / \mathrm{mL}$ actinomycin D (transcriptional inhibitor, Act D) prior to FSH $(10 \mathrm{ng} / \mathrm{mL})$ treatment. Data are shown as the mean \pm SEM. No less than three independent experiments were performed. Oneway ANOVA or Student's $t$-test is used for statistical analyses. ${ }^{*} P<0.05$ and ${ }^{* *} P<0.01$; ns, not significant

inhibitor, could block the effect of FSH on HMGCR transcription (Fig. 6j).

Moreover, to elucidate whether the suppression of FSH could decrease hepatic HMGCR expression, we performed experiments in mouse models in which FSH signaling was blocked. Our results showed that transcription and expression of SREBP-2 and HMGCR was significantly blocked in FSHAb-treated mice compared with the IgG-treated mice (Supplementary information, Figs. S7 and S8). In addition, we also found that the transcription and expression levels of hepatic HMGCR were significantly decreased in the Fshr ${ }^{-1-}$ mice in comparison with their $\mathrm{Fshr}^{+/+}$littermates (Supplementary information, Fig. S9). These data suggest that FSH regulates the transcription of hepatic $H M G C R$, inducing de novo cholesterol biosynthesis in the liver.
We also investigated the effect of FSH in LDL receptor deficiency (Idlr-/-) mice. The results showed that the serum TC and LDL-C levels, as well as the liver contents of TC and FC, were significantly elevated in the $\mathrm{FSH}$ treated Idlr-/- mice. The expressions of the mRNAs and proteins of HMGCR and SREBP-2 were also increased with FSH injection. Moreover, the cholesterol accumulation induced by FSH can also be blocked by the specific anti-FSH antibody (FSHAb) previously employed (Supplementary information, Fig. S10).

SREBP-2/FoxO1 is indispensable for the regulation of HMGCR by FSH

CREB and SREBP-2 are the two primary transcription regulators for HMGCR. $^{25,35}$ We showed that FSH upregulated SREBP-2 
158

maturation (SREBP-(N)), but not CREB phosphorylation ( $p$-CREB), in a time-dependent manner (Fig. 7a). As CREB is a CAMP-response element binding protein, we also detected CAMP levels and no significant changes were found after FSH stimulation, which was consistent with previous results (Fig. 7b). Using a luciferase reporter assay, we found that FSH could significantly increase the HMGCR luciferase activity in cells transfected with plasmids that contained the CRE and SRE sites (hHMGCR/luc) or the mutated CRE site (muCRE-hHMGCR/luc) but not in cells transfected with plasmids that contained the mutated SRE site (muSRE-hHMGCR/ luc) (Fig. 7c). Moreover, the effect of FSH on HMGCR disappeared when SREBP-2 was suppressed by shRNA (Fig. 7d). These results strongly indicate an indispensable role of SREBP-2 in the regulation of HMGCR by FSH.

We also detected the regulation of SREBP-2 by FSH and found that FSH increased the nascent transcription of SREBP-2 using the run-on assay (Fig. 7e). In addition, FSH also increased the activity of the SREBP-2 promoter-driven luciferase reporter (SREBP-2/luc) in vivo and in vitro (Fig. 7f, g). There are 2 IRE sites (IRE1 and IRE2) in the SREBP-2 promoter that can be recognized by the Forkhead box protein $\mathrm{O} 1$ (FoxO1), which is a negative regulator of SREBP-2 transcription. ${ }^{36}$ Thus, we mutated IRE sites within the SREBP-2 gene promoter to verify the role of FSH in the regulation of SREBP2 (Fig. 7f). The results showed that mutations in either IRE1 or IRE2 could completely block the effect of FSH on SREBP-2 (Fig. 7f), suggesting the essential roles of both IRE1 and IRE2. The chromatin immunoprecipitation (ChIP) assay showed that FSH reduced the binding capacity of FoxO1 to the SREBP-2 promoter (Fig. 7h). Western blotting results showed that the transfer of FoxO1 from the plasma into the nucleus was significantly attenuated in FSH-treated mice (Fig. 7i). These data demonstrated that FSH modulated the transcription of SREBP-2 by inhibiting the binding of FoxO1 to the SREBP-2 promoter.

FSH activates hepatic cholesterol biosynthesis via $\mathrm{Gi} 2 \mathrm{\alpha} / \beta$ arrestin2/Akt pathway

It is widely accepted that the function of FSH is mainly mediated through FSHRs, which belong to the G protein-coupled receptors (GPCRs). The FSHR in ovarian cells couples with a Gsa and elevates CAMP. ${ }^{20}$ Although the cAMP content did not change in the hepatocytes, Akt phosphorylation (p-Akt) was significantly increased with FSH treatment (Fig. 8a). In addition, the ability of FSH to increase the HMGCR expression and SREBP-2 maturation was prevented by a specific p-Akt inhibitor (MK2206) or PI3K inhibitor (LY294002) (Fig. 8b-d).

To find the specific type of Ga isoform that participated in the process of $\mathrm{FSH}$-mediated cholesterol regulation, we used a group of siRNAs to silence the corresponding $\mathrm{Ga}$ isoforms (Fig. 8e). Intriguingly, only when Gi2a was silenced, the effects of FSH on HMGCR transcription and expression, as well as on SREBP-2 maturation, were interrupted (Fig. 8f, g). $\beta$-arrestins, including $\beta$ arrestin1 (ARRB1) and $\beta$-arrestin 2 (ARRB2), were ubiquitously expressed and participated in many metabolic processes through activation of Akt. ${ }^{37-39}$ In our study, we genetically established stable $A R R B 1$ and ARRB2 gene-knockdown hepatic cell lines, and the data demonstrated that when ARRB2 was knocked down, the effects of FSH on Akt phosphorylation and HMGCR expression were interrupted (Fig. $8 \mathrm{~h}, \mathrm{i}$ ). These results suggested that hepatocytes possess Gi2a-coupled FSHRs to activate the $\beta$ arrestin-2/Akt/SREBP-2 pathway, resulting in enhanced expression of HMGCR and increased cholesterol biosynthesis after FSH stimulation.

\section{DISCUSSION}

This is the first report proving that blocking FSH signaling by ablating the Fshr gene or using an epitope-specific antibody against FSH could reduce cholesterol accumulation in the body.
This study identified that elevated FSH alone leads to an unfavorable circulating TC disturbance by regulating hepatic cholesterol biosynthesis in the liver, which could be reversed by blocking FSH signaling (Fig. 9).

Given that FSH levels increase while estrogen levels decrease during menopause, and considering the association between estrogen and cholesterol metabolism, ${ }^{40}$ we could not determine whether FSH regulates cholesterol levels without excluding the effects of estrogen. To study the effects of FSH per se in an epidemiological investigation, we first recruited both premenopausal and peri-menopausal females. The serum E2 levels were similar in the two groups, however, the FSH levels were significantly increased in the peri-menopausal group. Furthermore, we adjusted the data by considering the effects of E2 and other confounders (e.g., age, BMI) using multivariate models ${ }^{2,41}$ and ultimately found that the serum FSH levels were independently and positively correlated with the cholesterol levels.

We noted that there was no striking difference in the serum cholesterol level between the human pre- and peri-menopausal groups. This lack of difference may occur because the serum FSH level during the peri-menopausal stage increases gradually but does not reach the peak, ${ }^{1}$ as a result, cholesterol may change gradually. During the post-menopausal stage, when the serum FSH level reaches a peak, women tend to have higher cholesterol levels. ${ }^{1,42}$ Although the increase in the mean level of serum cholesterol was not very striking, we found that the prevalence of hypercholesterolemia had significantly increased in the perimenopausal subjects. Thus, it is necessary to test the serum lipid profile for aging women in as early as the peri-menopause stage in clinical settings.

In our project on animal experiments, we generated mouse models that mimic mice in peri-menopausal stage by eliminating the influence of estrogen deficiency. The surgical OVX mice were supplemented with exogenous E2 to maintain similar E2 levels as compared with Sham-operated mice. Under these conditions, the levels of endogenous FSH were also stable due to E2 feedback at the pituitary gland. Using this approach, we were able to control the levels of FSH by administering exogenous FSH without altering the E2 levels.

The liver plays a pivotal role in lipid metabolism. ${ }^{33}$ Cholesterol metabolism includes cholesterol biosynthesis, uptake, conversion, transport and esterification. ${ }^{23}$ By screening molecules related to cholesterol metabolism based on mRNA and protein expression variations, we found FSH regulated cholesterogenesis. By measuring the rate of hepatic de novo cholesterol biosynthesis and the expression of its rate-limiting enzyme, HMGCR, we demonstrated the direct effects of FSH on cholesterol biosynthesis. The work by Song et al reports that FSH may interact with its receptors in hepatocytes and reduce LDLR levels, which subsequently attenuates the endocytosis of LDL-C, resulting in an elevated circulating LDL-C level. ${ }^{11}$ Different results observed in two laboratories may lie in different experimental models.

Our study demonstrated that FSH increased the cholesterol level via regulating HMGCR. Statins, as inhibitors of HMGCR, are widely used in the treatment of hypercholesterolemia. ${ }^{43}$ Thus, statins should be very effective for treating menopause-associated cholesterol increase. Several clinical trials have provided evidence for the efficacy and safety of statins usage in post-menopausal women, such as simvastatin, pravastatin, fluvastatin or lovastatin. $^{44-47}$ In addition to cholesterol disorder, menopause is associated with bone loss, ${ }_{16}^{16}$ enhanced visceral adiposity, ${ }^{48}$ and neuropsychiatric disorders. ${ }^{49}$ Previous studies showed that blocking FSH prevented bone loss, ${ }^{17,18,32}$ and reduced body fat, ${ }^{17}$ and in this study we proved that blocking FSH signaling inhibited hepatic cholesterol biosynthesis and reduced the serum cholesterol level. As a result, targeting FSH signaling might provide multifaceted benefits. 
a
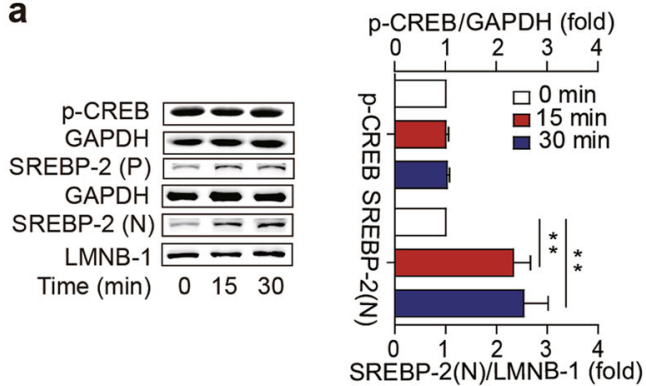

b

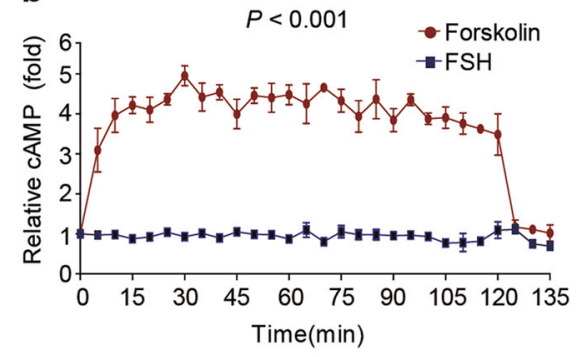

C
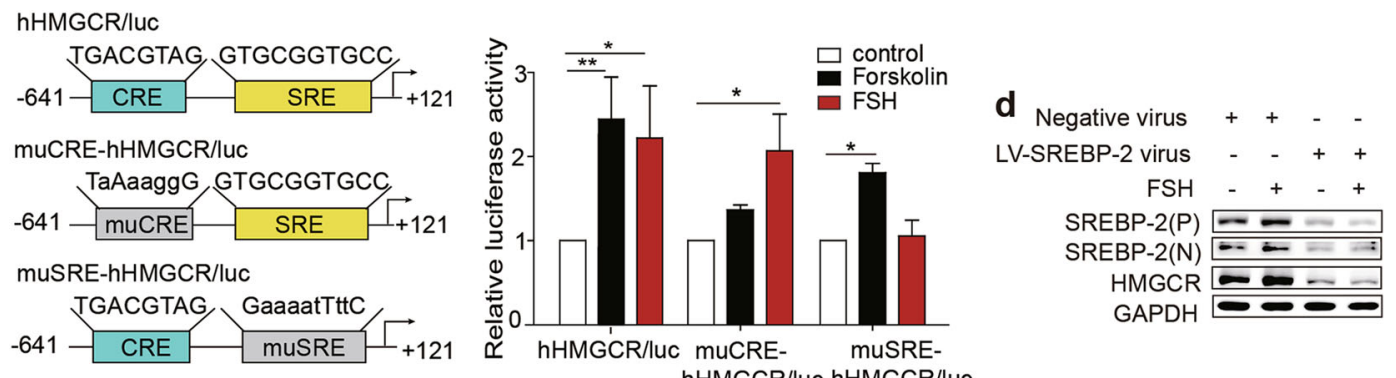

e

f

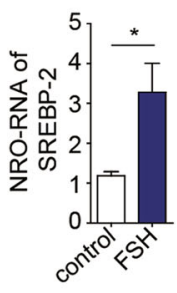

SREBP-2/luc

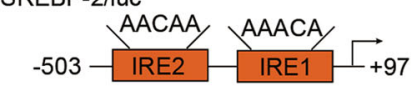

muIRE1-SREBP-2/luc

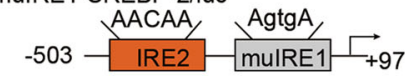

mulRE2-SREBP-2/luc
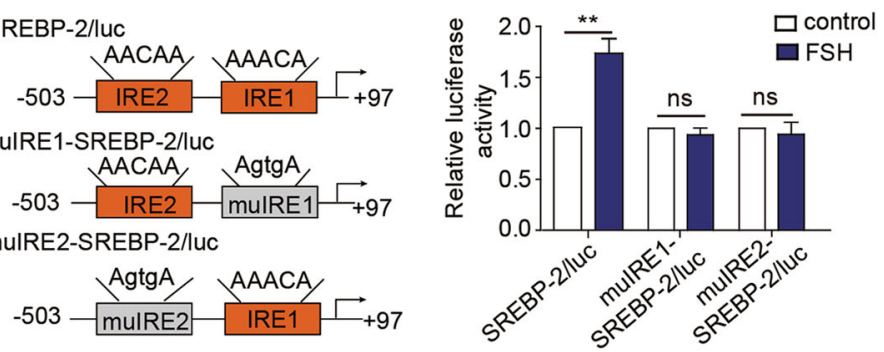

g
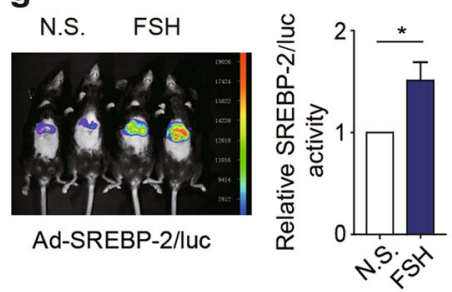

h

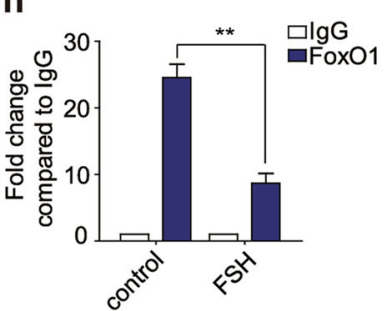

i

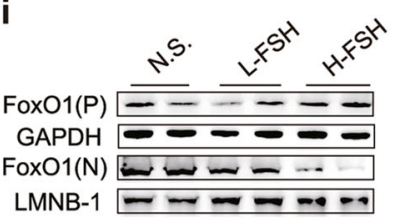

Ad-SREBP-2/luc

$4.2 s^{\lambda}$

Fig. 7 SREBP-2/FoxO1 is indispensable for the regulation of HMGCR by FSH. a Representative immunoblotting of SREBP-2 and p-CREB in HepG2 cells treated with FSH $(10 \mathrm{ng} / \mathrm{mL})$ for different times. b The cAMP levels in HepG2 cells treated with Forskolin $(50 \mu \mathrm{M})$ or FSH (10 $\mathrm{ng} / \mathrm{mL})$ for different times. Forskolin $(50 \mu \mathrm{M})$ was used as a positive control. $P<0.001$. c (Left) The human reporter vector containing CRE and SRE sites in the HMGCR promoter (hHMGCR/luc) and vectors containing CRE motif mutant (muCRE-hHMGCR/luc) or SRE motif mutant (muSREhHMGCR/luc) were designed. (Right) HepG2 cells were transfected with these vectors for $24 \mathrm{~h}$ and were subsequently treated with FSH (10 ng/ $\mathrm{mL})$, the relative luciferase activity was assayed. Forskolin $(50 \mu \mathrm{M})$ was used as a positive control. d Western blotting analyses of SREBP-2 and HMGCR in HepG2 cells transfected with SREBP-2 shRNA virus (LV-SREBP-2 virus) or with negative control virus followed by FSH (10 ng/mL) treatment. e Assay for the nascent transcription of SREBP-2 in the HepG2 cell line by run-on assay. $\mathbf{f}$ (Left) The SREBP-2 reporter construct containing IRE1 and IRE2 sites (SREBP-2/luc) and constructs with IRE motif mutants (muIRE1 or muIRE2) were made. (Right) HepG2 cells were transfected with these constructs for $24 \mathrm{~h}$ and were subsequently treated with $\mathrm{FSH}(10 \mathrm{ng} / \mathrm{mL})$, the relative luciferase activity was assayed. g Live imaging of hepatic SREBP-2-luciferase activity in OVX + E2 mice injected with Ad-SREBP-2/luc via tail vein and then solvent (N.S.) or FSH (30 IU $/ \mathrm{kg})(n=3$ per group). Live imaging of luciferase activity was normalized to coinfected RSV $\beta$-gal reporter activity in the liver. Bar graph is shown as the mean \pm SEM. $\mathbf{h}$ HepG 2 cells were incubated with FSH $(10 \mathrm{ng} / \mathrm{ml})$ for $12 \mathrm{~h}$ and then ChIP-qPCR analysis was performed using antiFoxO1 antibody. The data were normalized to the corresponding IgG. $\mathbf{i}$ Western blotting results showing the protein level of FoxO1 in the cytoplasm and the nuclei in OVX mouse model ( $n=9$ per group). For all panels in this figure, data are represented as the mean \pm SEM for no less than three independent experiments. $\mathrm{N}$ represents nuclear protein; $\mathrm{P}$ represents cytoplasmic protein. For $\mathbf{b}$, generalized estimating equation (GEE) is used for statistical analysis. For $\mathbf{a}, \mathbf{c}, \mathbf{e}-\mathbf{h}$, One-way ANOVA or Student's $t$-test is used for statistical analysis. ${ }^{*} P<0.05$ and ${ }^{* *} P<$ $0.01 ; \mathrm{ns}$, not significant 
a

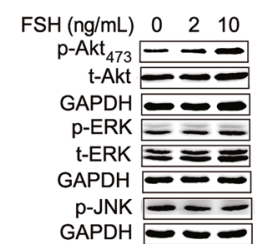

b

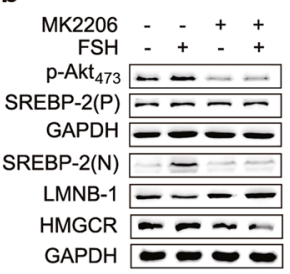

c

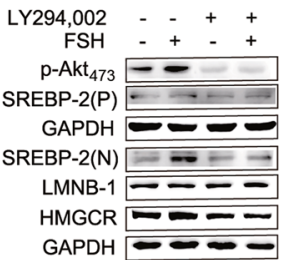

d

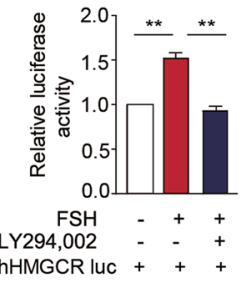

e

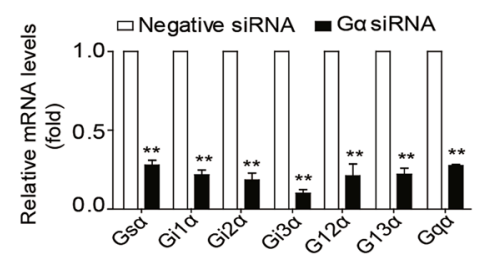

f

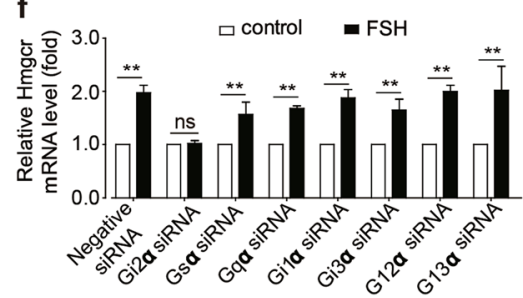

g

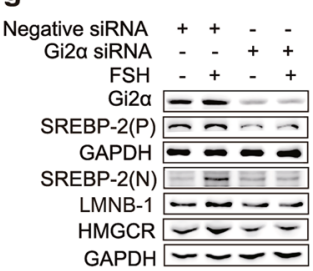

h

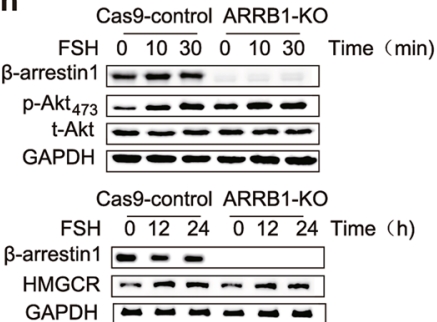

i

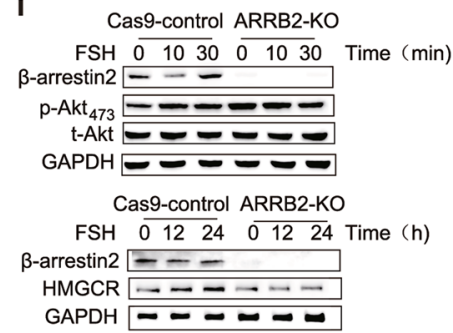

Fig. $8 \mathrm{FSH}$ activates hepatic cholesterol biosynthesis via the Gi2 $\alpha / \beta$-arrestin2/Akt pathway. a Representative immunoblotting of phosphorylated (p-) Akt, ERK, or JNK levels in response to FSH stimulation (10 ng/mL). b Representative immunoblotting of $p$-Akt, SREBP-2 and HMGCR in HepG2 cells treated with MK2206 $(10 \mu \mathrm{M})$, a p-Akt specific inhibitor, prior to FSH $(10 \mathrm{ng} / \mathrm{mL})$ treatment. c Representative immunoblotting of p-Akt, HMGCR and SREBP-2 in HepG2 cells treated with LY294002 (10 $\mu \mathrm{M})$, a PI3K inhibitor, prior to FSH (10 ng/mL) treatment. d The luciferase activity of HepG2 cells transfected with the hHMGCR/luc vector for $24 \mathrm{~h}$ and then treated with LY294002 (10 $\mu \mathrm{M})$ followed by FSH $(10 \mathrm{ng} / \mathrm{mL})$ treatment. e qPCR of $G \alpha$ proteins in HepG2 cells transfected with different siRNAs that suppress the corresponding $\mathrm{G} \alpha$ subunits. $\mathbf{f}$ siRNAs were used to suppress different $\mathrm{G} \alpha$ isoforms in HepG 2 cells, the effect of FSH on HMGCR mRNA levels was subsequently analyzed. Negative siRNA was used as a negative control. g After the knockdown of Gi2a by siRNA in HepG2 cells, the protein levels of SREBP-2 and HMGCR were detected by Western blotting following treatment with FSH (10 $\mathrm{ng} / \mathrm{mL}$ ). Representative immunoblotting of Akt phosphorylation and HMGCR expression in ARRB1 (h) or ARRB2 (i) gene-knockdown HepG2 cell lines. For all panels in this figure, data are shown as the mean \pm SEM for no less than three independent experiments. $\mathrm{N}$ represents nuclear protein; $\mathrm{P}$ represents cytoplasmic protein. One-way ANOVA or Student's $t$-test is used for statistical analysis. ${ }^{* *} P<0.01$; ns, not significant

The liver is the key metabolic organ that regulates glucose and lipid metabolism. ${ }^{50}$ It has been proven that there is a cross-talk between glucose and lipid metabolism. ${ }^{51,52}$ In this study, we found that FSH upregulated hepatic cholesterol biosynthesis via promoting AKT-dependent FoxO1 phosphorylation. Our team previously found that FSH enhanced gluconeogenesis without effecting insulin, dependent on AMP-activated protein kinase (AMPK) phosphorylation via GRK2 in the liver. ${ }^{53}$ FoxO1 could integrate insulin signaling to hepatic glucose and lipid metabolism. ${ }^{54-56}$ AMPK is a major cellular energy sensor and a master regulator of metabolic homeostasis, involved in both glucose and lipid metabolism. ${ }^{57-59}$ As FSH regulates the phosphorylation of FoxO1 and AMPK, FSH-induced hyperglycemia and hypercholesterolemia are quite likely to be mutually influential. However, to what extent could the effects on glucose metabolism interfere with or affect the results obtained here on lipid metabolism needs further investigation.

In conclusion, this study characterized a novel role of $\mathrm{FSH}$, independent of estrogen, as a potential regulator of hepatic cholesterol biosynthesis. We show for the first time that blocking FSH signaling could significantly reduce cholesterol biosynthesis in the liver, thereby ameliorating high serum level of cholesterol. This finding may be helpful in devising therapy strategies, as well as in exploring novel hormone-based therapies, for hypercholesterolemia during the menopausal transition ${ }^{7}$ or in hypogonadism. ${ }^{60}$

\section{MATERIALS AND METHODS}

Epidemiological investigation

Epidemiological design. This study was a cross-sectional study conducted in Ningyang County in the Shandong Province of China in 2014. Registered Chinese residents aged $\geq 40$ years who had lived in their current residence for at least 5 years were invited, by telephone or door-to-door visits, to participate in a screening examination at the recruitment site. All female participants provided an overnight fasting blood sample, underwent a medical examination and were instructed to complete a self-reported questionnaire, including detailed questions regarding menopausal status. The ethics committee of the Shandong Provincial Hospital approved the study, and the procedures were conducted in accordance with the Declaration of Helsinki. Written informed consent was obtained from each participant prior to data collection.

We enrolled female participants who experienced natural menstrual states, based on the following criteria: (1) providing vital data, such as age, sex, sex hormone levels, and serum lipid profiles; (2) no presence of complications or conditions that affect menopausal status or lipid metabolism, such as uterectomy, bilateral ovariectomy, premature ovarian failure, history of irregular menstruation during premenopause, pregnancy, lactation, psychiatric illness, acute cardiovascular or cerebrovascular disease, chronic respiratory disease, familial hypercholesterolemia, 


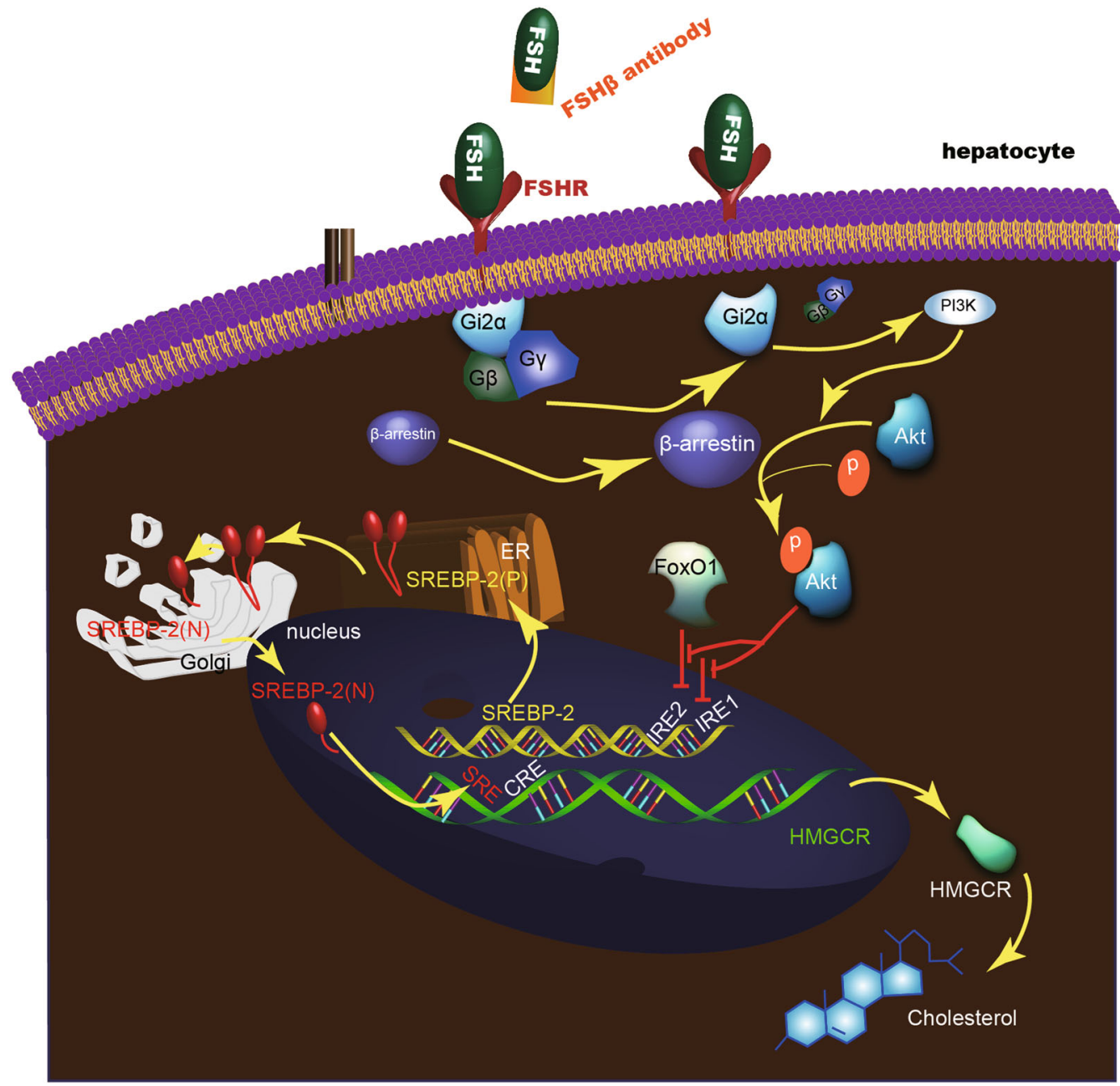

Fig. 9 The schematic model depicts the regulation process of de novo cholesterol biosynthesis by FSH in the liver. FSH couples with FSHR on the hepatocyte surface and then activates Gi2 $\alpha$, which promotes the process of Akt phosphorylation by PI3K. Moreover, ARRB2 ( $\beta$-arrestin2) is involved in the regulation of Akt phosphorylation by FSH. The phosphorylated Akt decreases the transfer of FoxO1 from the cytoplasm into the nucleus, which reduces its inhibition of SREBP-2 transcription. As a gatekeeper of cholesterol biosynthesis, mature SREBP2 promotes the transcription and expression of HMGCR, the rate-limiting enzyme in cholesterol biosynthesis. In summary, elevated FSH induces hepatic de novo cholesterol biosynthesis

malignant tumors, hepatic (alanine aminotransferase (ALT) and/or aspartate aminotransferase levels above $40 \mathrm{U} / \mathrm{L}$ ), or renal (evaluated glomerular filtration rate below $60 \mathrm{~mL} / \mathrm{min}$ per $1.73 \mathrm{~m}^{2}$ as calculated using the simplified Modification of Diet in Renal Disease equation) dysfunctions; (3) no use of medications that affect the natural state of menopause or lipid metabolism, including estrogen, progesterones, androgens, glucocorticoids, statins, fibrates, thyroid hormones, anti-thyroid drugs, nonsteroidal anti-inflammatory drugs, $\beta$-adrenoceptor blockers, or thiazide diuretics, over the preceding three months. As a result, 154 pre-menopausal and 124 peri-menopausal female subjects were eligible for investigation in our study.

Data collection. The determination of menopausal status was based on the responses to questions regarding menstrual irregularity and amenorrhea in the self-reported questionnaire. Peri-menopause was defined as the presence of menses within the past 3 months with a decrease in cycle predictability in the year preceding examination or 3-11 months of amenorrhea. To investigate the association between serum FSH and cholesterol levels independent of E2, we further selected peri-menopausal subjects with the presence of menses within the past 3 months with a decrease in cycle predictability in the year preceding examination because their E2 levels have been reported to be similar to those in pre-menopause. ${ }^{1,61}$ For the pre-menopausal participants, venipuncture was scheduled on days 2-5 of a spontaneous menstrual cycle to evaluate sex hormone levels. For the peri-menopausal participants, fasting serum samples were randomly obtained. ${ }^{62}$

All investigators successfully completed a training program that was standardized and scripted for all investigators to minimize the interrater variability. Blood samples were collected from all participants between 8:00 AM and 10:00 AM after a minimum of 10-h fasting. ${ }^{1}$ All blood samples were immediately placed on ice, processed within 30 minutes and preserved at $-80^{\circ} \mathrm{C}$ until detections, except for the fasting plasma glucose and hemoglobin A1c $(\mathrm{HbA1c})$ levels, which were measured within $2 \mathrm{~h}$. The examinations of the blood samples were all completed at the clinical laboratory of the Shandong Provincial Hospital. The intraassay and inter-assay coefficients of variation were kept below $5 \%$ for all the parameters.

Trained investigators obtained information on age, sex and other essential data from the standardized questionnaire. ${ }^{63}$ The weight and height were measured in kilograms and centimeters, respectively, and body mass index (BMI) was calculated by dividing the weight (kilograms) by the square of the height 
(square meters). The waist circumference was measured in centimeters. The blood pressure was measured three times for each individual with an electronic sphygmomanometer (HEM7117; Omron). There was an interval of $3 \mathrm{~min}$ between each measurement for each participant, and the mean value of the three measurements was calculated. The methods for determining weight, height, waist circumference, and blood pressure were similar to those used in the national survey of the prevalence of diabetes in 2007. ${ }^{64}$ Chemiluminescent methods (Cobas E601; Roche, Basel, Switzerland) were used to measure female sex hormones, including FSH and E2. The serum lipid profiles, plasma glucose level, $\mathrm{HbA} 1 \mathrm{c}$ level, as well as the parameters of hepatic and renal functions were determined using the BECKMAN Chemistry Analyzer AU5800 System (Beckman Coulter, Tokyo, Japan).

Hypercholesterolemia is defined as TC $\geq 6.2 \mathrm{mmol} / \mathrm{L}$ and/or LDL$\mathrm{C} \geq 4.1 \mathrm{mmol} / \mathrm{L}^{65,66}$

\section{Animals and treatment}

Ovariectomized mice. The animals were housed in a temperaturecontrolled room $\left(24^{\circ} \mathrm{C}\right)$ with a $12 \mathrm{~h}$ light, $12 \mathrm{~h}$ dark cycle. For ovary removal surgery, 9-week-old female C57BL/6 mice (Charles River Laboratory, Inc.) were administered general anesthesia and subjected to either a sham operation (Sham) or bilateral ovariectomy $(\mathrm{OVX}) .^{11}$ The serum levels of estrogen and FSH were tested after the operation. The OVX mice were supplemented with or without estradiol valerate (Bayer). Similar estrogen level of the former $(\mathrm{OVX}+\mathrm{E} 2)$ group was maintained as compared with the Sham group.

FSH treatment. The OVX $+\mathrm{E} 2$ mice received solvent (N.S.) or different doses of recombinant FSH (Gonal-F, Serono, Switzerland) via intraperitoneal injection. Based on different treatments, the groups were named as OVX + estradiol (E2), OVX + E2 + low-dose FSH (L-FSH; $15 \mathrm{IU} / \mathrm{kg}$ body weight per day) and OVX + E2 + highdose FSH (H-FSH; $30 \mathrm{lU} / \mathrm{kg}$ body weight per day). ${ }^{11}$

GnRHa treatment. The method for the treatment with GnRHa (Triptorelin Acetate Injection, Ferring) was conducted as previously described. ${ }^{11}$ The OVX + E2 mice received an intraperitoneal injection of solvent (N.S.) or GnRH analog (GnRHa; $25 \mu \mathrm{g} / \mathrm{kg}$ body weight per day). GnRHa leads to pituitary desensitization and the inhibition of pituitary FSH secretion. ${ }^{11,29}$

Anti-FSH $\beta$ antibody. FSH consists of two dissimilar subunits, the a and $\beta$ subunits. ${ }^{30}$ The FSH $\beta$ is responsible for the interaction with FSHR. $^{31}$ Mice received intraperitoneal injections of either anti$\mathrm{FSH} \beta$ antibody (MedixMab cat. \#6602, BiosPacific, Emeryville, CA) or mouse IgG at $100 \mu \mathrm{g} / \mathrm{d}$ daily. ${ }^{18,32}$ All OVX + E2 mice were injected with FSH or normal saline (N.S.) following the daily administration of anti-FSH $\beta$ antibody or mouse IgG for 4 weeks. Regarding another mouse model, the wild-type mice received antibody or lgG for 8 weeks. ${ }^{17}$

Injection of shRNA. Nine-week-old female C57BL/6 J mice were housed at $24^{\circ} \mathrm{C}$ in a $12 \mathrm{~h}$ light-dark cycle and humidity-controlled (60\%) environment. A synthetic Fshr shRNA, based on the high degree of hepatotropism of adenovirus5, was designed and synthesized by GENECHEM (Shanghai, China). The target sequence used for the mouse Fshr was 5'-TACCCAACTTGCATGAAAT-3', which was confirmed to effectively inhibit the expression of the FSH receptor in a mouse hepatic cell line, NCTC1469, prior to in vivo use (data not shown). The delivery of shRNA was performed via the tail vein through a high-volume injection according to the method of Fafalios et al. ${ }^{67}$ using $2 \times 10^{8}$ PFU of virus per 20 grams of body weight. These mice were designated as the Fshr shRNA virus group. For the tail-vein injection, the shRNA virus was administered in $0.5 \mathrm{~mL}$ (PBS) for the experimental group. The control mice were injected with a corresponding volume of negative adenovirus and were named the negative virus group. The procedure was repeated two additional times. Five days after the mice were ovariectomized, we supplied the mice with an estradiol valerate-supplemented diet. Two weeks before euthanasia, the mice from each group were injected with FSH or normal saline (N.S.).

Fshr knockout mice. Fshr- mice (WuHan Kangweisda Gene Technology, Inc, China) were from C57BL/6 J backgrounds. The 4week-old $\mathrm{Fshr}^{-1-}$ mice were fed with a $0.26 \%$ estradiol valerate (Bayer)-supplemented diet to maintain normal estrogen levels. Nineweek-old $\mathrm{Fshr}^{-/}$mice and their $\mathrm{Fshr}^{+/+}$littermates received ovary removal surgery, following estrogen supplementation. When the serum E2 levels achieved stability, the treatment with recombinant FSH (Gonal-F, Serono, Switzerland) was conducted by daily intraperitoneal injection for 2 weeks. In another mouse model, in order to explore the regulation of FSH on cholesterol during the post-menopausal stage, the $\mathrm{Fshr}^{-/}$mice and their $\mathrm{Fshr}^{+/+}$littermates received an ovariectomy operation with no other treatments.

Mice fed with High-cholesterol diet. Seven-week-old female wildtype mice and Fshr knockout mice (estrogen-supplemented) were fed with a normal-cholesterol diet (NC) or high-cholesterol diet (HC) (4\% cholesterol diet) for 20 weeks. The mice were sacrificed after fasting for approximately $8 \mathrm{~h}^{68}$

Ldlr knockout mice. Nine-week-old Idlr-/- mice were shamoperated (Sham) or ovariectomized (OVX). The estrogen (E2) level of the OVX mice were subsequently maintained at the normal level by exogenous estrogen supplementation and were injected with solvent (normal saline, N.S.) or exogenous FSH (H-FSH, 30 IU/ $\mathrm{kg}$ body weight per day) for 2 weeks.

Isolation and culture of primary mouse hepatocytes Mouse hepatocytes were isolated from the C57BL/6 or Fshr knockout mice as previously described. ${ }^{69}$

\section{Cell culture}

The human HepG2 cell line was obtained from the Type Culture Collection of the Chinese Academy of Sciences, Shanghai, China. These cells were grown in MEM medium supplemented with $10 \%$ FBS at $37^{\circ} \mathrm{C}$ with $5 \% \mathrm{CO}_{2}$. CHO cell line, as the positive control, was grown in DMEM/F12 medium supplemented with $10 \%$ FBS at $37^{\circ} \mathrm{C}$ with $5 \% \mathrm{CO} 2$.

\section{Genetic manipulation of ARRBs}

The CRISPR/Cas9 system was used for cellular genetic manipulation to generate a genetically stable gene knockdown cell line. In brief, HepG2 cells were cultured to $70-80 \%$ confluence and then transfected with the lentiviral vector Lenti-Cas9-EGFP. Puromycin was used for selecting a stable Cas9 expression strain. HepG2 cells with a stable expression of Cas9 (described as the Cas9-control in our research) were selected, subcultured with puromycin, and subsequently used for target gene knockdown. SgRNAs of human ARRB1 (GenBank: NC_000011) and ARRB2 (GenBank: NC_000017) were transfected into Cas9 control cells to obtain ARRB $1 / 2$ gene knockdown cell lines (referred to as ARRB1/2-KO), which specifically did not express the respective protein of $\beta$-arrestin-1 or $\beta$-arrestin- 2 . The sequences of the sgRNAs are listed as follows: ARRB1, 5'TTCTTGTCCTCGGGGGCCGG-3', and ARRB2, 5'-CCTGTTCATCGCCACCTACC $-3^{\prime}$. Western blotting analysis was performed to verify the levels of proteins encoded by the targeted genes.

Serum hormone measurements

The levels of serum E2 and FSH were measured using commercial immunoassay kits (Estradiol sensitive ELISA, Demeditec DE4399; 
FSH (Rodent) ELISA Kit, Abnova KA2330). Absorbance at $450 \mathrm{~nm}$ was determined using an ELISA microtiter plate reader (BioTeklnc., Winooski, VT, USA).To calculate the hormone concentration, a standard curve was constructed by plotting a graph of the absorbance of each reference standard.

\section{VLDL production rate}

To measure the VLDL production rate, overnight-fasted mice were injected i.p. with $1 \mathrm{~g} / \mathrm{kg}$ BW poloxamer 407 in PBS. Blood samples were collected immediately prior to injection and at 1, 2 and 3hours following injection. Cholesterol concentration was measured enzymatically. ${ }^{70}$

In-situ hybridization (ISH) assay

To optimize an ISH assay for FSHR, we first employed a panel of samples, human ovaries, previously analyzed for FSHR expression by $\mathrm{qPCR}$, as previously described. ${ }^{71}$ For this analysis, we validated our ISH assay on 2 positive samples for FSHR, with matched qPCR data. Following the confirmation of our method, we used ISH to analyze paraffin sections from human ovaries and livers.

Competitive FSH-FSHR binding assay

The competition assay for detecting the specific binding of FSH and FSHR was performed as previously described. ${ }^{72}$

iTRAQ labeling and NanoLC-MS/MS analysis

Whole protein samples were isolated using a standard protocol. The protein concentrations were assayed with a BCA protein assay kit (Bio-Rad, Richmond, CA, USA). For the proteomic analysis, 100 $\mu \mathrm{g}$ of protein from each sample was denatured with a denaturing buffer (0.5 M Tris- $\mathrm{HCl}, 2.75 \mathrm{mM}$ EDTA, $6 \mathrm{M}$ Guanidine- $\mathrm{HCl}$, adjusted to $\mathrm{pH} 8.1 \pm 0.1)$ and $1.0 \mathrm{M}$ DTT solution. The denatured proteins were alkylated with $1.0 \mathrm{M}$ lodoacetamide. After being desalted with $25 \mathrm{mM}$ of ammonium bicarbonate using a Microcon YM-10 (UFC501096, Millipore), the alkylated proteins were digested with 1:25 (w/w) trypsin at $37^{\circ} \mathrm{C}$ overnight (V511A, Promega). The tryptic peptides were then labeled with the iTRAQ Reagents 4-plex Kit (AB Sciex) according to the manufacturer's protocol. The details related to iTRAQ labeling were defined as previously reported. ${ }^{73}$

The MS analysis was performed using a NanoLC system (NanoLC-415 Ultra Plus, Eksigent) equipped with a Triple TOF 5600 Plus mass spectrometer (AB SCIEX). The functional annotation of the detected proteins was performed using the $\mathrm{GO}$ database. Functional categories within proteins that were significantly enriched relative to 3020 human plasma proteins were determined using the GO Tree Machine's algorithm. For a given GO category, the relative enrichment of the genes that encoded the proteins detected in HDL3 relative to all reference genes in that category were calculated. A cutoff value of $P<$ 0.0001 was used to report a functional category as significantly overrepresented. The results were confirmed by repeating the analysis using the Expression Analysis Systematic Explorer algorithm. The EASE uses a variant of the 1-tailed Fisher's exact probability test to determine an EASE score, which ranks overrepresented GO processes.

The cellular localization of the identified proteins was further analyzed based on the information available from the Gene Ontology (GO) and the Human Protein Reference Database (HPRD). Biological function classifications and signaling pathway analysis were performed with the tools in DAVID Bioinformatics Resources 2008 and the Kyoto Encyclopedia of Genes and Genome (STRING) database, respectively.

Suppression of SREBP-2 using siRNA

Short hairpin RNAi (shRNA) targeting the human SREBP-2 gene was designed and generated using stable lentivirus expression vectors at Shanghai GeneChem, China. The sequence of the shRNA against human SREBP-2 was 5'-CACCTGGACTCTTAAGAAA-3'.
Suppression of $G a$ subunits using siRNA

The siRNAs targeting $\mathrm{Ga}$ subunits were obtained from Thermo Fisher. The transfections were performed as previously described. ${ }^{25}$

Intracellular TC determination

Cholesterol was extracted from cells using a Cholesterol assay Kit (Applygen Technologies, Beijing, China) according to the manufacturer's instruction, and the cholesterol content was normalized by the corresponding protein content.

Nuclear run-on (NRO) RT-qPCR

The SREBP-2 and HMGCR transcriptional activities were measured by nuclear run-on RT-qPCR. ${ }^{74}$ Briefly, hepatocytes were treated with or without FSH for $24 \mathrm{~h}$; then, cell nuclei were isolated and NRO transcription was performed in the presence of bromouridine. Labeled nascent transcripts were purified by immunoprecipitation, and the transcript levels were determined by reversetranscription quantitative PCR (RT-qPCR).

Plasmid construction and Luciferase reporter assay

The -641 to +121 region that contained the CRE and SRE elements of human HMGCR (Gene ID: 3156) promoters was amplified from human genomic DNA template and inserted into a pGL4.15 empty vector (Promega), referred to as human HMGCR/ luc. This region with either a mutated CRE site (TGACGTAG to TaAaaggG) or a mutated SRE site (GTGCGGTGCC to GaaaatTttC) was inserted into the equivalent site of the pGL4.15. After transfection with a corresponding plasmid for $24 \mathrm{~h}$ following the manufacturer's instructions, HepG2 cells were treated with FSH for an additional $6 \mathrm{~h}$. The cells were subsequently harvested, and the luciferase activity was measured using a dual-luciferase reporter assay system (Promega). pRL-TK was used as a control to normalize the luciferase activity.

For the SREBP-2 (Gene ID: 6721) gene promoter analysis, we cloned the short promoter $(-503$ to $+97 \mathrm{bp}$ relative to the transcription start site) that contained IRE1 and IRE2 into the pGL4.10 vector (Promega). AAACA sequence was mutated to AgtgA sequence for IRE1 site of SREBP-2 promoter. AACAA sequence was mutated to AgtgA sequence for IRE2 site of SREBP-2 promoter.

Luciferase reporter analysis in vivo

Live imaging experiments were performed as previously described. ${ }^{75}$ Briefly, OVX mice were transfected with adenoviruses, HMGCR-Luc and RSV $\beta$-gal by tail-vein injection. Five days after the delivery of the adenovirus, the mice were imaged under ad libitum feeding conditions. Prior to imaging, the mice were intraperitoneally injected with $50 \mathrm{mg} / \mathrm{kg}$ pentobarbital and 100 $\mathrm{mg} / \mathrm{kg}$ sterile firefly D-luciferin (Promega). The mice were imaged with the LB983 Imaging System and analyzed with Living Image software (Berthold).

\section{Assay of HMGCR activity}

Hepatic microsomes were prepared as previously described using a differential ultracentrifugation method. ${ }^{76}$ The concentration of microsomes was determined by the BCA method. The HMGCR activity was accessed using the HPLC-MS/MS method as previously described. ${ }^{77}$

Measurement of de novo Cholesterol Biosynthesis

Mice were injected with $20 \mu \mathrm{Ci}\left[1{ }^{14} \mathrm{C}\right]$-sodium acetate (PerkinElmer, Waltham, MA) as previously described. ${ }^{78}$ The mice were sacrificed 20 minutes after injection, and $50 \mathrm{mg}$ of the liver was rinsed in ice-cold phosphate-buffered saline. The $\left[1-{ }^{14} \mathrm{C}\right]$-acetate content within the lipid fraction of each tissue was calculated from the measured disintegrations/minute $(\mathrm{dpm})$. The results were adjusted by the hepatic whole protein level. The de novo 
cholesterol biosynthesis was expressed as the amount of $\left[1-{ }^{14} \mathrm{C}\right]-$ acetate incorporated into sterols per gram of protein in the liver tissue.

\section{GloSensor cAMP assay}

The Glosensor plasmid was transfected into the HepG2 cells using Lipofectamine 3000 (Invitrogen). Twenty-four hours later, the cells were plated on 96 -well plates at a density of 20,000 cells per well. The cells were maintained in culture medium for an additional 22 $\mathrm{h}$ and were then incubated with $100 \mu \mathrm{l}$ of CO2-independent medium with $2 \% \mathrm{v} / \mathrm{v}$ GloSensor ${ }^{\mathrm{TM}}$ CAMP Reagent (Promega) and $10 \%$ FBS for two hours. The cAMP signal was monitored on a luminescence counter (Berthold LB942). ${ }^{79}$

\section{Chromatin immunoprecipitation (ChIP) assay}

The ChIP assay was performed using a ChIP assay kit according to the manufacturer's instructions (Merck). Briefly, the HepG2 cells were treated with $1 \%$ formaldehyde to cross-link the histones and genomic DNA. Cell lysates were prepared, and chromosomal DNA was sonicated to obtain average sizes between 200 and $1000 \mathrm{bp}$. The chromatin was incubated and precipitated with antibodies against FoxO1 or normal rabbit lgG (Proteintech) as controls at $4^{\circ}$ C overnight. A $230 \mathrm{bp}$ DNA fragment that contained the FoxO1 binding site of the SREBP-2 promoter was PCR-amplified for 45 cycles using $2 \mu \mathrm{l}$ of DNA as a template. The PCR products were analyzed by real-time PCR using a Roche LightCycler 480 Sequence Detection System.

\section{Immunofluorescence for frozen sections}

Frozen sections of mouse livers were fixed in $95 \%$ ethanol for $5 \mathrm{~s}$. Experiments were performed as previously described ${ }^{80}$ using antiFSHR antibody (1:150, Proteintech) and goat anti-rabbit lgG (1:100, Santa (ruz). Specimens were imaged using a super-resolution optic microscope (N-SIM, Nikon).

Total, nuclear and cytoplasmic protein extraction

For total protein extraction, the liver tissue samples and cells were homogenized in RIPA lysis buffer that contained 1\% PMSF and 1\% phosphatase inhibitor cocktail according to the manufacturer's protocol. For nuclear and cytoplasmic protein extraction, samples were prepared using NE-PER Nuclear and Cytoplasmic Extraction Reagents (Pierce). The protein concentration was measured using the BCA method.

RNA isolation and quantitative real-time PCR

Total RNA from tissue was isolated using Trizol reagent (Takara, Tokyo, Japan) according to the manufacturer's protocol. For the mRNA expression analysis, cDNA was synthesized using reverse transcriptase kits (RT) (Takara), following the manufacturer's protocol. Quantitative real-time PCR (qRT-PCR) analysis was performed in duplicate using LC480 (Roche, Mannheim, Germany) according to the instructions as previously described. ${ }^{68}$

\section{Western blotting}

Equal amounts of protein from different samples were separated in a $10 \%$ SDS-PAGE gel, then the samples were transferred from the gel to polyvinylidene difluoride membranes (Millipore). Following incubation for $1 \mathrm{~h}$ with $5 \%$ non-fat milk in TBST, the membranes were incubated overnight at $4{ }^{\circ} \mathrm{C}$ with anti-FSHR (1:1000, Abcam, and Proteintech), anti-HMGCR (1:1000, Abcam), anti-LDLR (1:200, Abcam), anti-CYP7A1 (1:200, Abcam), anti-ABCA1 (1:200, Abcam), anti-SRB1 (1:8000, Novus), anti-ACAT2 (1:1000, Proteintech), anti-p-CREB (1:1000, CST), anti-SREBP-2 (1:1000, designed by ChinaPeptides Co. Ltd.), and anti-Gi2a (1:1000, Proteintech) antibodies. Following the primary antibodies, the membranes were incubated with the corresponding secondary antibodies at a 1:5000 dilution for $1 \mathrm{~h}$ at room temperature. Immune complexes were detected using enhanced chemiluminescence reagents (Millipore). The same membrane was re-incubated with anti-GAPDH or anti-LMNB-1 antibodies. The GAPDH protein was used as a loading control for total and cytoplasmic proteins, and LMNB-1 was the control for nuclear proteins.

\section{$\mathrm{RT}^{2} \mathrm{PCR}$ array}

Gene expression profiling was performed in a 96-well plate with an $\mathrm{RT}^{2}$ Profiler PCR Array system (Qiagen) according to the manufacturer's instructions to examine the expression of 84 cholesterol metabolism-related genes. The real-time PCR was performed using a 40-cycle 2-step PCR protocol on a Light Cycler 480 (Roche).

\section{Statistical analysis}

The continuous variables data are presented as mean \pm standard deviation, mean \pm standard error or median (interquartile range, if non-normal distribution). Differences between two groups were compared using the Student's $t$-test, the Mann-Whitney U-test, or Generalized estimating equation (if non-normal distribution). Differences among multiple groups were compared using oneway analysis of variance (followed by a multiple comparison test for subgroups using Least Significant Difference or Dunnett's T3 tests). Multivariable linear regression model was used to analyze the association between FSH and cholesterol levels, with adjustment for estradiol levels and previously reported potential confounders. $^{2,41}$ Two-tailed $P$ values less than 0.05 were considered statistically significant. The analyses were performed using SPSS version 22.0 for Windows (Chicago, IL, USA).

Study approval

The animal studies were reviewed and approved by the Animal Ethics Committee at Shandong Provincial Hospital, China. For the epidemiological study, all subjects provided written informed consent. The studies on human samples were reviewed and approved by the Ethics Committee of Shandong Provincial Hospital. The study protocol conformed to the ethical guidelines of the 1975 Declaration of Helsinki and was approved by the Ethics Committee of Shandong Provincial Hospital.

\section{ACKNOWLEDGEMENTS}

We thank Prof. Yingli Lu (Shanghai JiaoTong University School of Medicine, China) for the assistance in the measurement of de novo cholesterol biosynthesis. Great thanks to Prof. Jinpeng Sun and Prof. Xiao Yu (Shandong University School of Medicine, China) for their technical assistance referring to $G$ proteins and $\beta$-arrestins in this project. We thank Shengfeng Wang (Department of Epidemiology and Biostatistics, Peking University Health Science Center, China) for providing professional instructions in statistical analyses. We are grateful to Prof. Yongsheng Chang (Chinese Academy of Medical Sciences and Peking Union Medical College) for his suggestions in writing the manuscript. And great thanks to Prof. Shucun Qin and his team (Taishan Medical University, China). This work was supported by grants from the National Natural Science Foundation (81870607, 81670796, 81430020, 81230018, 81300644 and 31640020 ) and National Key R\&D Program (SQ2017YFSF090203) of China.

\section{AUTHOR CONTRIBUTIONS}

Y.J.G. and Y.F.S. performed most of the experiments, analyzed and interpreted data and wrote the manuscript. T.B. helped to construct the Fshr ${ }^{-1-}$ mouse model and design some primers. H.Q.Z., M.Z., Z.S.Y., and L.L. conducted and analyzed the epidemiological data. M.S.Z. and W.B.C. performed the bioinformatic analysis. J.L. and Z.H.Z. provided the human liver samples. Z.L.Y. and M.C. provided the human ovarian samples. Q.X.W. and X.Y.L. helped to analyze the pathology results. M.X.S. and Q.H.L. helped to maintain the animal models. H.L.Y., and L.F.Z., performed some of the in vitro experiments. Q.W., C.X.Y., X.Y.Q., Y.L.L., and Y.J.L. helped with some of the in vivo experiments. Z.H., X.H., and L.W. helped to write the manuscript. L.G. and J.J.Z supervised the project and wrote the manuscript. 


\section{ADDITIONAL INFORMATION}

Supplementary information accompanies this paper at https://doi.org/10.1038/ s41422-018-0123-6.

Competing interests: The authors declare no competing interests.

\section{REFERENCES}

1. Randolph, J. J. F. et al. Change in follicle-stimulating hormone and estradiol across the menopausal transition: effect of age at the final menstrual period. $J$. Clin. Endocrinol. Metab. 96, 746-754 (2011).

2. Yang, W. et al. Serum lipids and lipoproteins in Chinese men and women. Circulation 125, 2212-2221 (2012)

3. Wenger, N. K. Clinical characteristics of coronary heart disease in women: emphasis on gender differences. Cardiovasc. Res. 53, 558-567 (2002).

4. van Beresteijn, E. C. H. et al. Perimenopausal increase in serum cholesterol: a 10year longitudinal study. Am. J. Epidemiol. 137, 383-392 (1993).

5. Rossouw, J. E. et al. Risks and benefits of estrogen plus progestin in healthy postmenopausal women: principal results from the Women's Health Initiative randomized controlled trial. JAMA 288, 321-333 (2002).

6. Randolph, J. F. et al. The value of follicle-stimulating hormone concentration and clinical findings as markers of the late menopausal transition. J. Clin. Endocrinol. Metab. 91, 3034-3040 (2006).

7. Matthews, K. A. et al. Are changes in cardiovascular disease risk factors in midlife women due to chronological aging or to the menopausal transition? J. Am. Coll. Cardiol. 54, 2366-2373 (2009).

8. Zhou, J. L. et al. Serum lipid profile changes during the menopausal transition in Chinese women: a community-based cohort study. Menopause 17, 997-1003 (2010).

9. Randolph, J. J. F. et al. Change in estradiol and follicle-stimulating hormone across the early menopausal transition: effects of ethnicity and age. J. Clin Endocrinol. Metab. 89, 1555-1561 (2004).

10. Green, J. S. et al. The effects of exercise training on abdominal visceral fat, body composition, and indicators of the metabolic syndrome in postmenopausal women with and without estrogen replacement therapy: The HERITAGE family study. Metabolism 53, 1192-1196 (2004).

11. Song, Y. et al. Follicle-stimulating hormone induces postmenopausal dyslipidemia through inhibiting hepatic cholesterol metabolism. J. Clin. Endocrinol. Metab. 101, 254-263 (2016)

12. Warner, M. \& Gustafsson, J.-A. On estrogen, cholesterol metabolism, and breast cancer. N. Engl. J. Med. 370, 572-573 (2014).

13. Manson, J. E. et al. Estrogen plus progestin and the risk of coronary heart disease. N. Engl. J. Med. 349, 523-534 (2003).

14. Sairam, M. R. in Encyclopedia of Reproduction, (eds Knobiland, E. \& Niell, J. D.) 552-565 (Academic Press, Inc, New York, 1999).

15. Sowers, M. R. et al. Amount of bone loss in relation to time around the final menstrual period and follicle-stimulating hormone staging of the transmenopause. J. Clin. Endocrinol. Metab. 95, 2155-2162 (2010).

16. Sun, L. et al. FSH directly regulates bone mass. Cell 125, 247-260 (2006).

17. Liu, P. et al. Blocking FSH induces thermogenic adipose tissue and reduces body fat. Nature 546, 107 (2017).

18. Zhu, L. L. et al. Blocking antibody to the $\beta$-subunit of FSH prevents bone loss by inhibiting bone resorption and stimulating bone synthesis. Proc. Natl Acad. Sci. 109, 14574-14579 (2012).

19. Chu, M. C., Rath, K. M., Huie, J. \& Taylor, H. S. Elevated basal FSH in normal cycling women is associated with unfavourable lipid levels and increased cardiovascular risk. Hum. Reprod. 18, 1570-1573 (2003).

20. Conti, M. Specificity of the cyclic adenosine $3^{\prime}, 5^{\prime}$-monophosphate signal in granulosa cell function. Biol. Reprod. 67, 1653-1661 (2002).

21. Liu, X. M. et al. FSH regulates fat accumulation and redistribution in aging through the Gai/Ca2+/CREB pathway. Aging Cell 14, 409-420 (2015).

22. Qi, S. et al. HSP27 phosphorylation modulates TRAIL-induced activation of SrcAkt/ERK signaling through interaction with $\beta$-arrestin2. Cell. Signal. 26, 594-602 (2014).

23. $\min , \mathrm{H}$. K. et al. Increased hepatic synthesis and dysregulation of cholestero metabolism is associated with the severity of nonalcoholic fatty liver disease. Cell. Metab. 15, 665-674 (2012).

24. Bloch, K. The biological synthesis of cholesterol. Science 150, 19-28 (1965).

25. Tian, L. et al. A novel role for thyroid-stimulating hormone: up-regulation of hepatic 3-hydroxy-3-methyl-glutaryl-coenzyme a reductase expression through the cyclic adenosine monophosphate/protein kinase $\mathrm{A} /$ cyclic adenosine monophosphate-responsive element binding protein pathway. Hepatology $\mathbf{5 2}$ 1401-1409 (2010).
26. Pertusa, M., Morenilla-Palao, C., Carteron, C., Viana, F. \& Cabedo, H. Transcriptional control of cholesterol biosynthesis in schwann cells by axonal neuregulin 1. J. Biol. Chem. 282, 28768-28778 (2007).

27. Horton, J. D., Goldstein, J. L. \& Brown, M. S. SREBPs: activators of the complete program of cholesterol and fatty acid synthesis in the liver. J. Clin. Invest. 109, 1125-1131 (2002).

28. Faulds, M. H., Zhao, C., Wright, K. D. \& Gustafsson, J.-Å. The diversity of sex steroid action: regulation of metabolism by estrogen signaling. J. Endocrinol. 212, 3-12 (2012).

29. Demeestere, I. et al. Gonadotropin-releasing hormone agonist for the prevention of chemotherapy-induced ovarian failure in patients with lymphoma: 1-year follow-up of a prospective randomized trial. J. Clin. Oncol. 31, 903-909 (2013).

30. Jiang, X., Dias, J. A. \& He, X. Structural biology of glycoprotein hormones and their receptors: insights to signaling. Mol. Cell. Endocrinol. 382, 424-451 (2014).

31. Jiang, $X$. et al. Structure of follicle-stimulating hormone in complex with the entire ectodomain of its receptor. Proc. Natl Acad. Sci. 109, 12491-12496 (2012).

32. Zhu, L. L. et al. Blocking FSH action attenuates osteoclastogenesis. Biochem. Biophys. Res. Commun. 422, 54-58 (2012).

33. Scheja, L. \& Heeren, J. Metabolic interplay between white, beige, brown adipocytes and the liver. J. Hepatol. 64, 1176-1186 (2016).

34. Geelen, M. H., Gibson, D. \& Rodwell, V. Hydroxymethylglutaryl-CoA reductasethe rate-limiting enzyme of cholesterol biosynthesis. FEBS Lett. 201, 183-186 (1986).

35. $\mathrm{Ng}$, R. et al. Inhibition of microRNA-24 expression in liver prevents hepatic lipid accumulation and hyperlipidemia. Hepatology 60, 554-564 (2014).

36. Tao, R., Xiong, X., DePinho, R. A., Deng, C.-X. \& Dong, X. C. Hepatic SREBP-2 and cholesterol biosynthesis are regulated by $\mathrm{FoxO}_{3}$ and Sirt6. J. Lipid Res. 54 2745-2753 (2013).

37. Beaulieu, J. M. et al. An Akt/ $\beta$-arrestin 2/PP2A signaling complex mediates dopaminergic neurotransmission and behavior. Cell 122, 261-273 (2005).

38. Lefkowitz, R. J. \& Shenoy, S. K. Transduction of receptor signals by ß-arrestins. Science 308, 512-517 (2005).

39. Yang, F. et al. Phospho-selective mechanisms of arrestin conformations and functions revealed by unnatural amino acid incorporation and 19F-NMR. Nat. Commun. 6, 8202 (2015)

40. Pedram, A. et al. Estrogen reduces lipid content in the liver exclusively from membrane receptor signaling. Sci. Signal. 6, ra36-ra36 (2013).

41. Oka, R. et al. Relationships between alanine aminotransferase (ALT), visceral adipose tissue (AT) and metabolic risk factors in a middle-aged Japanese population. J. Atheroscler. Thromb. 21, 582-592 (2014).

42. Choi, Y. et al. Menopausal stages and serum lipid and lipoprotein abnormalities in middle-aged women. Maturitas 80, 399-405 (2015).

43. Grundy, S. M. HMG-CoA reductase inhibitors for treatment of hypercholesterolemia. N. Engl. J. Med. 319, 24-33 (1988).

44. Fak, A. S. et al. Effects of simvastatin only or in combination with continuous combined hormone replacement therapy on serum lipid levels in hypercholesterolaemic post-menopausal women. Eur. Heart J. 21, 190-197 (2000).

45. Lemay, A., Dodin, S., Turcot, L., Déchêne, F. \& Forest, J. C. Estrogen/progesterone replacement versus pravastatin and their sequential association in hypercholesterolemic postmenopausal women. Maturitas 40, 247-257 (2001).

46. Serruys, P. W. et al. A randomized placebo-controlled trial of fluvastatin for prevention of restenosis after successful coronary balloon angioplasty: final results of the fluvastatin angiographic restenosis (FLARE) trial. Eur. Heart J. 20, 58-69 (1999).

47. Bradford, R. H. et al. Efficacy and tolerability of lovastatin in 3390 women with moderate hypercholesterolemia. Ann. Intern. Med. 118, 850-855 (1993).

48. Thurston, R. C. et al. Gains in body fat and vasomotor symptom reporting over the menopausal transition: the Study of Women's Health Across the Nation. Am. J. Epidemiol. 170, 766-774 (2009).

49. Haring, B. et al. Cardiovascular disease and cognitive decline in postmenopausal women: results from the Women's Health Initiative Memory Study. J. Am. Heart Assoc. 2, e000369 (2013).

50. van den Berghe, G. in Journal of Inherited Metabolic Disease (eds R. A. Harkness, R J. Pollitt, \& G. M. Addison) 407-420 (Springer Netherlands, 1991).

51. Nakagawa, Y. \& Shimano, H. CREBH regulates systemic glucose and lipid metabolism. Int. J. Mol. Sci. 19, 1396 (2018).

52. Mota, M., Banini, B. A., Cazanave, S. C. \& Sanyal, A. J. Molecular mechanisms of lipotoxicity and glucotoxicity in nonalcoholic fatty liver disease. Metabolism 65, 1049-1061 (2016).

53. Qi, X. et al. Follicle-stimulating hormone enhances hepatic gluconeogenesis by GRK2-mediated AMPK hyperphosphorylation at Ser485 in mice. Diabetologia 61 1180-1192 (2018).

54. Li, Y. et al. A global perspective on FOXO1 in lipid metabolism and lipid-related diseases. Prog. Lipid Res. 66, 42-49 (2017). 
55. Matsumoto, M., Han, S., Kitamura, T. \& Accili, D. Dual role of transcription factor FoxO1 in controlling hepatic insulin sensitivity and lipid metabolism. J. Clin. Invest. 116, 2464-2472 (2006).

56. Lee, S. \& Dong, H. H. FoxO integration of insulin signaling with glucose and lipid metabolism. J. Endocrinol. 233, R67-R79 (2017).

57. Smith, B. K. \& Steinberg, G. R. AMP-activated protein kinase, fatty acid metabolism, and insulin sensitivity. Curr. Opin. Clin. Nutr. Metab. Care 20, 248-253 (2017).

58. Gopoju, R., Panangipalli, S. \& Kotamraju, S. Metformin treatment prevents SREBP2-mediated cholesterol uptake and improves lipid homeostasis during oxidative stress-induced atherosclerosis. Free Radic. Biol. Med. 118, 85-97 (2018).

59. Zhang, B. B., Zhou, G. \& Li, C. AMPK: an emerging drug target for diabetes and the metabolic syndrome. Cell. Metab. 9, 407-416 (2009).

60. Oppenheim, D. S., Greenspan, S. L., Zervas, N. T., Schoenfeld, D. A. \& Anne Klibanski, M. Elevated serum lipids in hypogonadal men with and without hyperprolactinemia. Ann. Intern. Med. 111, 288-92 (1989).

61. Derby, C. A. et al. Lipid changes during the menopause transition in relation to age and weight: the Study of Women's Health Across the Nation. Am. J. Epidemiol. 169, 1352-1361 (2009).

62. Nagaraj, N. et al. Complement proteins and arterial calcification in middle aged women: cross-sectional effect of cardiovascular fat. The SWAN Cardiovascular Fat Ancillary Study. Atherosclerosis 243, 533-539 (2015).

63. Luepker, R. V., Evans, A., McKeigue, P. \& Reddy, K. S. in Cardiovascular Survey Methods (World Health Organization, Geneva, 2004).

64. Yang, W. et al. Prevalence of diabetes among men and women in China. N. Engl. J. Med. 362, 1090-1101 (2010)

65. Guidelines for the management of dyslipidaemia in chinese adults. Chin. Circ. J. 31, 937-953 (2016).

66. Expert Panel on, D. Evaluation, and Treatment of High Blood Cholesterol in Adults. Executive summary of the third report of the national cholesterol education program (ncep) expert panel on detection, evaluation, and treatment of high blood cholesterol in adults (adult treatment panel iii). JAMA 285, 2486-2497 (2001).
67. Fafalios, A. et al. A hepatocyte growth factor receptor (Met)-insulin receptor hybrid governs hepatic glucose metabolism. Nat. Med. 17, 1577 (2011).

68. Li, Y. et al. A novel role for CRTC2 in hepatic cholesterol synthesis through SREBP2. Hepatology 66, 481-497 (2017).

69. Zhang, X. et al. Thyroid-stimulating hormone decreases HMG-CoA reductase phosphorylation via AMP-activated protein kinase in the liver. J. Lipid Res. 56, 963-971 (2015).

70. Aryal, B. et al. Absence of ANGPTL4 in adipose tissue improves glucose tolerance and attenuates atherogenesis. JCl Insight 3, e97918 (2018).

71. Prensner, J. R. et al. The long noncoding RNA SChLAP1 promotes aggressive prostate cancer and antagonizes the SWI/SNF complex. Nat. Genet. 45, 1392 (2013).

72. Abe, E. et al. TSH is a negative regulator of skeletal remodeling. Cell 115, 151-162 (2003).

73. Wang, Y. et al. Tomato nuclear proteome reveals the involvement of specific E2 ubiquitin-conjugating enzymes in fruit ripening. Genome Biol. 15, 548 (2014).

74. Roberts, T. C. et al. Quantification of nascent transcription by bromouridine immunocapture nuclear run-on RT-qPCR. Nat. Protoc. 10, 1198 (2015).

75. Dentin, R. et al. Insulin modulates gluconeogenesis by inhibition of the coactivator TORC2. Nature 449, 366 (2007)

76. Honda, A. et al. Differences in hepatic levels of intermediates in bile acid biosynthesis between Cyp27-/- mice and CTX. J. Lipid Res. 42, 291-300 (2001).

77. Honda, A. et al. Highly sensitive assay of HMG-CoA reductase activity by LC-ESIMS/MS. J. Lipid Res. 48, 1212-1220 (2007).

78. Li, T. et al. Overexpression of cholesterol 7a-hydroxylase promotes hepatic bile acid synthesis and secretion and maintains cholesterol homeostasis. Hepatology 53, 996-1006 (2011).

79. Wang, H. M. et al. A stress response pathway in mice upregulates somatostatin level and transcription in pancreatic delta cells through Gs and $\beta$-arrestin 1. Diabetologia 57, 1899-1910 (2014).

80. Song, Y. et al. Thyroid-stimulating hormone regulates hepatic bile acid homeostasis via SREBP-2/HNF-4a/CYP7A1 axis. J. Hepatol. 62, 1171-1179 (2015). 